\title{
Polyvector deformations in eleven-dimensional supergravity
}

\author{
Kirill Gubarev@* and Edvard T. Musaev $\circledast^{\dagger}$ \\ Moscow Institute of Physics and Technology, Institutskii pereulok 9, Dolgoprudny 141700, Russia
}

(Received 8 December 2020; accepted 3 March 2021; published 26 March 2021)

\begin{abstract}
We consider 3- and 6-vector deformations of 11-dimensional supergravity backgrounds of the form $M_{5} \times M_{6}$ admitting at least three Killing vectors. Using flux formulation of the $\mathrm{E}_{6(6)}$ exceptional field theory, we derive (sufficient) conditions for the deformations to generate a solution. In the group manifold case, these generalizations of the classical Yang-Baxter equation for the case of r-matrices with three and six indices are shown to reproduce those obtained from exceptional Drinfeld algebra for $\mathrm{E}_{6(6)}$. In general, we see an additional constraint, which might be related to higher exceptional Drinfeld algebras.
\end{abstract}

DOI: 10.1103/PhysRevD.103.066021

\section{INTRODUCTION}

Vacua of string theory understood as a perturbative formulation of the nonlinear two-dimensional sigma-model are known to be represented by a vast landscape of 10-dimensional manifolds equipped by various gauge fields: Kalb-Ramond 2-form and Ramond-Ramond $p$-form gauge fields. In the full nonperturbative formulation, one finds that the space of string vacua is mostly populated by 11-dimensional manifolds, and 10-dimensional backgrounds represent points with a small string coupling constant (see [1] for a more detailed review). A set of string vacua possesses a huge amount of various symmetries that prove useful in better understanding of its structure. In particular, one finds T-duality symmetries: Abelian [2,3], non-Abelian [4], and more generally, Poisson-Lie dualities [5-7], which are related to backgrounds that are indistinguishable from the perturbative string point of view. Nonperturbatively symmetries get enhanced to (Abelian) U-dualities, which can be understood as transformations related to toroidal backgrounds equivalent from the point of view of the membrane $[8,9]$. Certain progress toward defining non-Abelian generalization of U-dualities have been made recently in [10-14].

At the level of low-energy theory of background fields such duality symmetries appear as solution-generating transformations. More generally, one is interested in

\footnotetext{
*kirill.gubarev@phystech.edu; Also at the Institute for Experimental and Theoretical Physics.

'musaev.et@phystech.edu; Also at Kazan Federal University, Institute of Physics.

Published by the American Physical Society under the terms of the Creative Commons Attribution 4.0 International license. Further distribution of this work must maintain attribution to the author(s) and the published article's title, journal citation, and DOI. Funded by SCOAP ${ }^{3}$.
}

transformations that keep the string sigma-model in a consistent vacuum, however changing it in a controllable way. Particularly interesting examples are based on manifolds with an AdS factor known to be holographically dual to superconformal field theories. While in general, CFTs are an isolated point in the space of couplings corresponding to a fixed point of renormalization group flow, SCFTs belong to a family of theories connected by varying couplings. Adding exactly marginal operators to a theory will preserve a conformal symmetry at the quantum level and move the corresponding point in the space of couplings along the socalled a conformal manifold [15]. Certain progress in understanding of the structure of conformal manifold can be made by investigating the gravitational side of the AdS/CFT correspondence. Indeed, given a set of exactly marginal operators that deform a SCFT keeping it on a conformal manifold, there exists a family of dual AdS solutions related by the deformations of metric, dilaton, and $p$-form gauge fields. A well-known example is provided by $\beta$-deformations of $D=4 \mathcal{N}=4 \mathrm{SYM}$, whose gravity dual is a bi-vector Abelian (TsT) deformation along two of three $\mathrm{U}(1)$ isometry directions of $\mathbb{S}^{5}$ [16]. In a similar fashion considering $\mathrm{AdS}_{4} \times \mathbb{S}^{7}$, one is able to pick three $\mathrm{U}(1)$ directions of the seven-sphere to construct a trivector deformation of the ABJM theory. For a general formula for TsT transformations of gauge theories, see [17].

Generalizing the results known for TsT deformations, one naturally gets interested in bi- and tri-vector deformations along with a set of noncommuting Killing vectors. As the most symmetric example here, one finds deformations of two-dimensional sigma models preserving integrability, e.g., $\eta$-deformation of the Green-Schwarz superstring on $\mathrm{AdS}_{5} \times \mathbb{S}^{5}$ constructed in $[18,19]$. The choice of the Dynkin diagram for the corresponding superalgebra gives rise to the so-called ABF background [20], which solves equations of motion of generalized supergravity [21-23] or 
to normal supergravity backgrounds. To depart from backgrounds given by group manifolds and coset spaces, one generalizes the procedure and for a general deformation parametrized by a bi-vector $\Theta=r^{i j} k_{i} \wedge k_{j}$ obtains

$$
(g+b)^{-1}=(G+B)^{-1}+\Theta,
$$

where $G, B$ and $g, b$ are the metrices and the 2-form fields for the initial and deformed backgrounds, respectively [24-26]. Although at this level, the deformation is considered as a transformation of (generalized) supergravity solutions without a direct reference to two-dimensional sigma-models, both initial and deformed field configurations could be understood as consistent sigma-model backgrounds. Both the sigma-model (for coset spaces) and the field theory (for general manifolds with Killing vectors) approaches show that a deformed background is a solution of (generalized) supergravity equations when the r-matrix $r^{i j}$ satisfies classical Yang-Baxter equation (CYBE) $[27,28]$

$$
r^{k\left[i_{1}\right.} r^{i_{2}|l|} f_{k l}^{\left.i_{3}\right]}=0 .
$$

Here, $f_{i j}{ }^{k}$ are structure constants of the algebra of Killing vectors.

Notably, the transformation (1) appears essentially nonlinear only due to a bad choice of variables. It becomes a linear $\mathrm{O}(10,10)$ transformation when written in terms of generalized metric i.e., a representative of the coset $\mathrm{O}(10,10) / \mathrm{O}(1,9) \times \mathrm{O}(9,1)$. For this reason, deriving classical Yang-Baxter equation from the standard supergravity formalism faces huge technical difficulties and has been done in $[27,29]$ only in the second order in $\Theta$. The formalism of double field theory [30,31] appears more natural, with the generalized metric being the canonical variable, which allowed a full proof in [28] that CYBE is sufficient to end up with a solution. Moving to tri-vector deformations, one naturally employs the exceptional field theory formalism (ExFT) for precisely the same reasons: the generalized metric transforms linearly under deformations. The generalization of the deformation map (1) obtained in the formalism of SL(5) ExFT in [32] finds the same interpretation as an open-closed membrane map [33]. Examples of non-Abelian tri-Killing deformations based on the open-closed membrane map or equivalently a specially defined SL(5) transformation have been provided for the $\mathrm{AdS}_{4} \times \mathbb{S}^{7}$ background in [34].

One may naturally ask whether the condition that a trivector deformation generates a solution of supergravity is equivalent to some algebraic condition generalizing CYBE. One first notices that CYBE appears when deforming generators of a Manin triple $\left(T_{i}, T^{i}, \eta\right)$ representing a Drinfeld double algebra by r-matrix

$$
\begin{aligned}
T_{i} & \rightarrow T_{i}, \\
T^{i} & \rightarrow T^{i}+r^{i j} T_{j},
\end{aligned}
$$

and requiring the deformed generators to also form a Drinfeld double algebra. Similarly, deforming generators of an exceptional Drinfeld algebra (EDA) by tri- and sixvector tensors $\rho^{i_{1} \ldots i_{3}}, \rho^{i_{1} \ldots i_{6}}$ and restricting the deformed generators to form an EDA, one arrives at a set of conditions on $\rho$-tensors. The exceptional Drinfeld algebra based on the SL(5) group has been constructed in $[10,11]$ and for the group $\mathrm{E}_{6(6)}$, this has been done in [35]. Conditions on the 3 -vector deformation tensor $\rho^{i_{1} i_{2} i_{3}}$ derived for the SL(5) EDA in [10] are equivalent to the unimodularity condition

$$
\left.\rho^{k l\left[i_{1}\right.} f_{k l} l_{2}\right]=0 .
$$

This condition is the only one to appear in the SL(5) case due to the dimension $d=4$ of the internal manifold, which appears to be too small to embed 3-vector deformations. The situation is the same as for bi-vector deformations in dimension $d=3$, where one gets only the unimodularity condition.

In contrast, the deformations considered inside the $\mathrm{E}_{6(6)}$ EDA are subject to a nontrivial constraint that is supposed to generalize classical Yang-Baxter deformation. In this work, we investigate whether this condition is sufficient for a deformation to be a solution generating a transformation as for the bi-vector case. To this end, we start with providing a short review of the $\mathrm{E}_{6(6)}$ extended geometry in Sec. II. In Sec. III, we define a deformation map for $\mathrm{E}_{6(6)}$ generalized vielbein and investigate the transformation of generalized fluxes under the map. We find the generalized Yang-Baxter equation of [35] as a sufficient condition for the fluxes to stay undeformed. Also we observe an additional constraint whose algebraic interpretation is yet unknown.

\section{TRUNCATION OF 11D SUPERGRAVITY}

The bi-vector deformation of backgrounds of a nonlinear sigma-model is given by the nonlinear map (1), whose form cannot be called self-evident. Due to a nonlinear nature of the map explicit check that this is a solution generating a transformation is a highly nontrivial task, as it has been demonstrated in [27]. Choosing the correct representation of degrees of freedom allows turning the deformation map into a linear transformation. Hence, for bi-vector deformations, one organizes NS-NS fields into the generalized metric of the double field theory parametrizing the coset $O(d, d) / O(d) \times O(d)$ and the invariant dilaton, and R-R fields into an $O(d, d)$ spinor. In this case, the transformation becomes simply an $O(d, d)$ rotation, and showing its solution-generating nature becomes a straightforward task (see $[26,36,37]$ for more detail). 
Similarly, as it has been shown in [32] that tri-vector deformations of the 11-dimensional background can be defined as an SL(5) transformation of generalized metric of the corresponding exceptional field theory. Below, we show that for 3-vector deformations within the SL(5) theory to generate a solution unimodularity is sufficient. Hence, no generalized Yang-Baxter equation is generated both in the field theory and in the SL(5) EDA. Here, we consider the $\mathrm{E}_{6(6)}$ exceptional field theory that allows 3 - and 6-vector deformations and, according to the analysis of the exceptional Drinfeld algebra of [35], a nontrivial generalization of the classical Yang-Baxter equation exists.

A detailed presentation of the $\mathrm{E}_{6(6)}$ exceptional field theory can be found in [38-40]. The presentation includes bosonic and fermionic field content, supersymmetry transformations, full Lagrangian and truncations to the 11-dimensional, and Type IIB supergravities. For a review of the recent progress, see [41,42]. For our purposes here, we stress the following defining features of the theory:

(i) ExFT is an $\mathrm{E}_{d(d)}$-covariant background independent theory combining full 11-dimensional and Type IIB supergravities (no reduction);

(ii) field content is represented by tensors of GL(11-d) taking values in certain representations of the U-duality group;

(iii) section condition, restricting dependence of fields on the total $5+27$ coordinates is required. In what follows, we assume the standard solution of the section constraint leaving only dependence on $5+6$ coordinates.

Hence, for the purposes of this work, the exceptional field theory simply provides a convenient rewriting of degrees of freedom of the 11-dimensional supergravity, turning polyvector deformations into a linear map.

The construction of fields and the Lagrangian of the $\mathrm{E}_{6(6)}$ exceptional field theory starting from the $5+6$ split of the 11-dimensional supergravity is given in detail in [38]. To introduce notations and for further reference, we provide a brief overview of the setup. The bosonic field content of the 11-dimensional supergravity consists of the elfbein $\hat{E}_{\hat{\mu}}{ }^{\hat{}}$ and the 3-form potential $C_{\hat{\mu}_{1} \hat{\mu}_{2} \hat{\mu}_{3}}$. Keeping full dependence on all of the 11 coordinates $x^{\hat{\mu}}$, one splits the fields into tensors in 5-dimensions and organizes them into multiplets of $\mathrm{E}_{6(6)}$. For the latter, one has to follow the dualization prescription of [43]. Decomposing the 11-dimensional indices as $\hat{\mu}=(\mu, m), \hat{\alpha}=(\bar{\mu}, a)$, one parametrizes the elfbein in the following upper-triangular form

$$
\hat{E}_{\hat{\mu}}^{\hat{\alpha}}=\left(\begin{array}{cc}
e^{-\frac{1}{3}} g_{\mu}{ }^{\bar{\mu}} & A_{\mu}{ }^{m} e_{m}{ }^{a} \\
0 & e_{m}{ }^{a}
\end{array}\right),
$$

and redefines the fields arising from the 3 -form potential as

$$
\begin{aligned}
A_{m n k}= & C_{m n k}, \\
A_{\mu m n}= & C_{\mu m n}-A_{\mu}{ }^{k} C_{k m n}, \\
A_{\mu \nu m}= & C_{\mu \nu m}-2 A_{[\mu}{ }^{n} C_{\nu] m n}+A_{\mu}{ }^{n} A_{\nu}{ }^{k} C_{m n k}, \\
A_{\mu \nu \rho}= & C_{\mu \nu \rho}-3 A_{[\mu}{ }^{m} C_{\nu \rho] m}+3 A_{[\mu}{ }^{m} A_{\nu}{ }^{n} C_{\rho] m n} \\
& -A_{\mu}{ }^{m} A_{\nu}{ }^{n} A_{\rho}{ }^{k} C_{m n k} .
\end{aligned}
$$

The resulting fields

$$
\left\{g_{\mu}^{\bar{\mu}}, A_{\mu}{ }^{m}, e_{m}{ }^{a}, A_{m n k}, A_{\mu n k}, A_{\mu \nu k}, A_{\mu \nu \rho}\right\}
$$

transform appropriately under splitted 11-dimensional diffeomorphisms $\xi^{\hat{\mu}}=\left(\xi^{\mu}, \Lambda^{m}\right)$. To organize these into multiplets of $\mathrm{E}_{6(6)}$, one has to dualize all forms to the lowest possible rank. Hence, the two forms $A_{\mu \nu m}$ get dualized intro 1-forms and can be collected with $A_{\mu}{ }^{m}$ and $A_{\mu m n}$ into the vector $A_{\mu}{ }^{M}$ of ExFT transforming in the 27 of $\mathrm{E}_{6(6)}$; the 3-form $A_{\mu \nu \rho}$ after dualization contributes to the scalar coset. The 6-dimensional space gets extended to include coordinates corresponding to the winding modes of the M2- and M5-branes and is now parametrized by $\mathbb{X}^{M}$ transforming as the $\mathbf{2 7}$ under global U-duality transformations. Local coordinate transformations on such extended space are given by the so-called generalized Lie derivative

$$
\begin{aligned}
\mathcal{L}_{\Lambda} V^{M}= & \Lambda^{K} \partial_{K} V^{M}-V^{K} \partial_{K} \Lambda^{M} \\
& +10 d^{M K R} d_{N L R} V^{N} \partial_{K} \Lambda^{L}-\left(\lambda-\frac{1}{3}\right) V^{M} \partial_{K} \Lambda^{K},
\end{aligned}
$$

where $V^{M}$ denote the components of some generalized vector of weight $\lambda$ and $d^{M N K}$, and $d_{M N K}$ are the invariant tensors of $\mathrm{E}_{6(6)}$. For such defined transformations to form a closed algebra, one imposes the section constraint

$$
d^{M N K} \partial_{N} \bullet \partial_{M} \bullet=0,
$$

where the bullets denote any fields and their combinations. The above condition has two (maximal) inequivalent solutions corresponding to embeddings of the 11dimensional and Type IIB 10-dimensional supergravity. We will be working with the former, i.e., always take into account the decomposition of $\mathfrak{e}_{6(6)}$ irreps under its subalgebra $\mathfrak{g l}(6)$. For the coordinates $\mathbb{X}^{M}$, this reads

$$
\mathbb{X}^{M}=\left(x^{m}, x_{m n}, x^{\bar{m}}\right),
$$

where $m, n=\overline{1,6}, \bar{m}, \bar{n}=\overline{1,6}$. We refer to Appendix A for the used index notations and conventions and notice here that the barred indices label the same $\mathbf{6}$ of $\mathrm{E}_{6(6)}$, and these are distinguished from the unbarred small Latin indices for technical convenience. In what follows, we always assume 


$$
\partial_{\bar{m}} f=0, \quad \partial^{m n} f=0,
$$

for any field $f$ of the theory. Upon such decomposition, nonvanishing components of the symmetric invariant tensor can be written as

$$
\begin{aligned}
d^{\bar{n} n}{ }_{m_{1} m_{2}} & =\frac{1}{\sqrt{5}} \delta_{\left[m_{1}{ }^{n}\right.} \delta_{\left.m_{2}\right]}{ }^{n}, \\
d_{n_{1} n_{2} n_{3} n_{4} n_{5} n_{6}} & =\frac{1}{4 \sqrt{5}} \epsilon_{n_{1} n_{2} n_{3} n_{4} n_{5} n_{6},}, \\
d_{\bar{n} n}{ }^{m_{1} m_{2}} & =\frac{1}{\sqrt{5}} \delta^{\left[m_{1}\right.} \delta^{\left.m_{2}\right]_{n},}, \\
d^{n_{1} n_{2} n_{3} n_{4} n_{5} n_{6}} & =\frac{1}{4 \sqrt{5}} \epsilon^{n_{1} n_{2} n_{3} n_{4} n_{5} n_{6}} .
\end{aligned}
$$

Finally, the (bosonic) $\mathrm{E}_{6(6)}$ ExFT field content reads

$$
\left\{g_{\mu \nu}, \mathcal{M}_{M N}, A_{\mu}{ }^{M}, B_{\mu \nu M}\right\},
$$

where $g_{\mu \nu}$ is the metric of the external space, $\mathcal{M}_{M N}$ is the so-called generalized metric parametrizing the scalar coset, $A_{\mu}{ }^{M}$ is the generalized connection, and $B_{\mu \nu M}$ is set of two-forms.

It is convenient to turn from the generalized metric $\mathcal{M}_{M N}$ to generalized vielbeins $\mathcal{E}_{M}^{A}$ defined as

$$
\mathcal{M}_{M N}=\mathcal{E}_{M}{ }^{A} \mathcal{E}_{N}{ }^{B} \mathcal{M}_{A B},
$$

where $\mathcal{M}_{A B}$ is the constant matrix (unity, for concreteness). In terms of fields of the 11-dimensional supergravity, the generalized vielbein $\mathcal{E}_{M}^{A}$ and its inverse can be parametrized as follows

$$
\begin{aligned}
& \mathcal{E}_{M}^{A}=e^{\frac{1}{3}}\left[\begin{array}{ccc}
e_{m}^{a} & \frac{-1}{\sqrt{2}} e_{m}^{b} C_{b a_{1} a_{2}} & \frac{1}{2} e_{m}^{\bar{a}} U+\frac{1}{4} e_{n}^{\bar{a}} C_{m b_{1} b_{2}} V^{n b_{1} b_{2}} \\
0 & e_{\left[a_{1}\right.}^{m_{1}} e_{\left.a_{2}\right]}^{m_{2}} & -\frac{1}{\sqrt{2}} e_{b_{1}}^{m_{1}} e_{b_{2}}^{m_{2}} V^{b_{1} b_{2} \bar{a}} \\
0 & 0 & e^{-1} e_{\bar{m}}^{\bar{a}}
\end{array}\right], \\
& \mathcal{E}_{A}^{M}=e^{-\frac{1}{3}}\left[\begin{array}{ccc}
e_{a}^{m} & 0 & 0 \\
\frac{1}{\sqrt{2}} e_{a}^{n} C_{n m_{1} m_{2}} & e_{m_{1}}^{\left[a_{1}\right.} e_{m_{2}}^{\left.a_{2}\right]} & 0 \\
-\frac{e}{2} e_{a}^{\bar{m}} U+\frac{e}{4} e_{a}^{l} C_{l k n} V^{\bar{m} k n} & \frac{e}{\sqrt{2}} V^{a_{1} a_{2} \bar{m}} & e e_{\bar{m}}^{\bar{m}}
\end{array}\right] \text {. }
\end{aligned}
$$

where $e=\operatorname{det}\left(e_{m}^{a}\right)$ and the tri-vector $V^{m_{1} m_{2} m_{3}}=$ $\frac{e^{-1}}{3 !} \epsilon^{m_{1} m_{2} m_{3} n_{1} n_{2} n_{3}} C_{n_{1} n_{2} n_{3}}$. The scalar degree of freedom $U=$ $\frac{e^{-1}}{6 !} \epsilon^{n_{1} \ldots n_{6}} C_{n_{1} \ldots n_{6}}$ comes from dualization of the three-form $A_{\mu \nu \rho}$ and the procedure directly relating to these two can be found in [38]. Such defined generalized vielbein is a generalized vector of weight $\lambda=0$, which is necessary for the full Lagrangian of ExFT to be invariant

$$
\begin{aligned}
\mathcal{L}_{\Lambda} \mathcal{E}^{M}{ }_{A}= & \Lambda^{K} \partial_{K} \mathcal{E}^{M}{ }_{A}-\mathcal{E}^{K}{ }_{A} \partial_{K} \Lambda^{M} \\
& +10 d^{M K R} d_{N L R} \mathcal{E}^{N}{ }_{A} \partial_{K} \Lambda^{L}+\frac{1}{3} \mathcal{E}^{M}{ }_{A} \partial_{K} \Lambda^{K} .
\end{aligned}
$$

In this work, we focus only on the scalar sector of the theory, i.e., only on the fields entering the generalized vielbein. As in the SL(5) theory [34], consistent truncation requires to also keep track of determinant of the external metric $g_{\mu \nu}$. Below, we discuss the rescaling of the generalized metric that combines all these degrees of freedom and decouples them from the rest of the fields.

\section{A. Generalized flux formulation}

For simplification of further discussion, we consider only such backgrounds that can be presented as $M_{11}=M_{6} \times M_{5}$, where the internal metric $g_{m n}$, the 3 -form $C_{m_{1} m_{2} m_{3}}$, and the 6-form $C_{m_{1} m_{2} m_{3} m_{4} m_{5} m_{6}}$ do not depend on the external coordinates $y^{\mu}$. Also, we take $A_{\mu}{ }^{M}=0$ and $B_{\mu \nu M}=0$. Hence, we consider the following ansatz

$$
\begin{aligned}
g_{\mu \nu} & =g_{\mu \nu}\left(y^{\mu}, x^{m}\right), \quad \mathcal{M}_{M N}=\mathcal{M}_{M N}\left(x^{m}\right), \\
A_{\mu}{ }^{M} & =0, \quad B_{\mu \nu M}=0 .
\end{aligned}
$$

Since the external metric is a scalar of nonzero weight under the generalized Lie derivative and, as we discuss later, the deformations are given by $\mathrm{E}_{6(6)}$ transformations, the external metric transforms by rescaling. It is convenient to explicitly factor out the part of nonzero weight $e^{2 \phi}$ of the external metric and further restrict the coordinate dependence as $g_{\mu \nu}\left(y^{\mu}, x^{m}\right)=e^{-2 \phi\left(x^{m}\right)} e^{\frac{2}{9-d}} \bar{g}_{\mu \nu}\left(y^{\mu}\right)\left(d=6\right.$ for $\left.\mathrm{E}_{6(6)}\right)$. The factor $e^{-2 \phi\left(x^{m}\right)}$ is possible to combine with the generalized metric of ExFT $\mathcal{M}_{M N}$ to define

$$
\begin{aligned}
g_{\mu \nu}\left(y^{\mu}, x^{m}\right) & =e^{-2 \phi\left(x^{m}\right)} e^{\frac{2}{9-d} \bar{g}_{\mu \nu}\left(y^{\mu}\right),} \\
\mathcal{M}_{M N} & =e^{-2 \phi} e^{\frac{2}{9-d}} M_{M N} .
\end{aligned}
$$

Applied to the SL(5) theory, one has $d=4$, and the metric $M_{M N}$ will be precisely that of the truncated theory of [44]. Such rescaled generalized metrics $M_{M N} \in E_{6(6)} \times \mathbb{R}^{+}$and can be represented in terms of the generalized vielbein $E_{M}{ }^{A} \in E_{6(6)} \times \mathbb{R}^{+}$as usual

$$
M_{M N}=E_{M}^{A} E_{N}^{B} M_{A B} .
$$

In components, the generalized vielbein and its inverse read 


$$
E_{M}^{A}=e^{\phi}\left[\begin{array}{ccc}
e_{m}^{a} & \frac{-1}{\sqrt{2}} e_{m}^{b} C_{b a_{1} a_{2}} & \frac{1}{2} e_{m}^{\bar{a}} U+\frac{1}{4} e_{n}^{\bar{a}} C_{m b_{1} b_{2}} V^{n b_{1} b_{2}} \\
0 & e_{\left[a_{1}\right.}^{m_{1}} e_{\left.a_{2}\right]}^{m_{2}} & -\frac{1}{\sqrt{2}} e_{b_{1}}^{m_{1}} e_{b_{2}}^{m_{2}} V^{b_{1} b_{2} \bar{a}} \\
0 & 0 & e^{-1} e_{\bar{m}}^{\bar{a}}
\end{array}\right],
$$$$
E_{A}^{M}=e^{-\phi}\left[\begin{array}{ccc}
e_{a}^{m} & 0 & 0 \\
\frac{1}{\sqrt{2}} e_{a}^{n} C_{n m_{1} m_{2}} & e_{m_{1}}^{\left[a_{1}\right.} e_{m_{2}}^{\left.a_{2}\right]} & 0 \\
-\frac{e}{2} e_{a}^{\bar{m}} U+\frac{e}{4} e_{a}^{l} C_{l k n} V^{\bar{m} k n} & \frac{e}{\sqrt{2}} V^{a_{1} a_{2} \bar{m}} & e e_{\bar{a}}^{\bar{m}}
\end{array}\right] \text {. }
$$

One lists some useful relations: $g=\operatorname{det}\left(g_{\mu \nu}\right)=$ $\left(e^{-2 \phi} e^{\frac{2}{3}}\right)^{5} \bar{g}\left(y^{\mu}\right)=M^{-\frac{5}{27}} \bar{g}\left(y^{\mu}\right), \quad g_{\mu \nu}=M^{-\frac{1}{27}} \bar{g}_{\mu \nu} \quad M^{\frac{1}{2}}=E=$ $\operatorname{det}\left(E_{M}^{A}\right)=\left(e^{\phi} e^{-\frac{1}{3}}\right)^{27}, \quad \mathcal{M}^{\frac{1}{2}}=\mathcal{E}=\operatorname{det}\left(\mathcal{E}_{M}^{A}\right)=1, \mathcal{M}_{M N}=$ $M^{-\frac{1}{27}} M_{M N}$.

Due to rescaling, the generalized vielbeins $E^{M}{ }_{A}$ and $\mathcal{E}^{M}{ }_{A}$ transform with different weights $\lambda\left[E^{M}{ }_{A}\right]=\frac{1}{3}, \lambda\left[\mathcal{E}^{M}{ }_{A}\right]=0$ and hence, one has

$$
\begin{aligned}
\mathcal{L}_{\Lambda} E^{M}{ }_{A}= & \Lambda^{K} \partial_{K} E^{M}{ }_{A}-E^{K}{ }_{A} \partial_{K} \Lambda^{M} \\
& +10 d^{M K R} d_{N L R} E^{N}{ }_{A} \partial_{K} \Lambda^{L} .
\end{aligned}
$$

Dropping all terms in the full Lagrangian of the exceptional field theory that do not give contributions to the equations of motion of the generalized metric and $\operatorname{det} g_{\mu \nu}$ upon $A_{\mu}{ }^{M}=0, B_{\mu \nu M}=0$, one has

$$
g^{-\frac{1}{2}} \mathcal{L}=\mathcal{R}\left[g_{\mu \nu}\right]+\mathcal{L}_{s c}\left(\mathcal{M}_{M N}, g_{\mu \nu}\right)
$$

where $\mathcal{R}\left[g_{\mu \nu}\right]$ is the usual Ricci scalar, and the scalar potential is given by

$$
\begin{aligned}
\mathcal{L}_{\mathrm{sc}}( & \left.\mathcal{M}_{M N}, g_{\mu \nu}\right) \\
= & -\frac{1}{24} \mathcal{M}^{M N} \partial_{M} \mathcal{M}^{K L} \partial_{N} \mathcal{M}_{K L} \\
& -\frac{1}{2} \mathcal{M}^{M N} \partial_{M} \mathcal{M}^{K L} \partial_{L} \mathcal{M}_{N K}-\frac{1}{2} g^{-1} \partial_{M} g \partial_{N} \mathcal{M}^{M N} \\
& -\frac{1}{4} \mathcal{M}^{M N} g^{-2} \partial_{M} g \partial_{N} g-\frac{1}{4} \mathcal{M}^{M N} \partial_{M} g^{\mu \nu} \partial_{N} g_{\mu \nu}
\end{aligned}
$$

Upon rescaling as above, the Lagrangian can be written in the following simple form

$$
\mathcal{L}=\bar{g}^{\frac{1}{2}} M^{-\frac{1}{18}}\left(\mathcal{R}\left[\bar{g}_{\mu \nu}\right]+\tilde{\mathcal{L}}_{\mathrm{sc}}\left(M_{M N}\right)\right),
$$

where $\tilde{\mathcal{L}}_{\mathrm{sc}}\left(M_{M N}\right)$ is the same as in the case of the nonlinear realization of $\mathrm{E}_{6(6)}$ ExFT [45]. This is due to the fact that the generalized metric obtained in the nonlinear realization has the same rescaling symmetry as our truncated metrics $M_{M N}$.

Now, one applies the same logic as in [34], and notices that the equations of motion of the rescaled generalized metric are those coming from the scalar potential $\tilde{\mathcal{L}}_{\text {sc }}\left(M_{M N}\right)$ plus the "cosmological term" coming from the curvature scalar of the external space $R\left[\bar{g}_{\mu \nu}\right]$.

$$
\frac{\delta \tilde{\mathcal{L}}_{\mathrm{sc}}\left(M_{M N}\right)}{\delta M_{M N}}-\frac{1}{18} \tilde{\mathcal{L}}_{\mathrm{sc}}\left(M_{M N}\right) M^{M N}=\frac{1}{18} R\left[\bar{g}_{\mu \nu}\right] M^{M N}
$$

In what follows, we assume that these equations are satisfied for the undeformed background and search for conditions upon which the deformation does not spoil this.

In what follows, it proves convenient to turn to the socalled flux formulation of the scalar sector of the exceptional field theory and to rewrite the above Lagrangian in terms of generalized fluxes as in [46]. Indeed, written completely in terms of the components of fluxes $\mathcal{F}_{A B}{ }^{C}$, to be defined below, the equations of motion will be guaranteed to hold after the deformation if the latter preserves the flux components. Given the deformation is an $\mathrm{E}_{6(6)}$ transformation, this would simply be a requirement for the flux components to transform covariantly.

In [47], for the generalized fluxes of double field theory, this requirement has been equivalent to the classical YangBaxter equation. The same idea is applicable here. Hence, one defines

$$
\begin{aligned}
\mathcal{L}_{E_{A}} E^{M}{ }_{B}= & E^{K}{ }_{A} \partial_{K} E^{M}{ }_{B}-E^{K}{ }_{B} \partial_{K} E^{M}{ }_{A} \\
& +10 d^{M K R} d_{N L R} E^{N}{ }_{B} \partial_{K} E^{L}{ }_{A}=\mathcal{F}_{A, B}{ }^{C} E^{M}{ }_{C} \\
\mathcal{F}_{A, B}{ }^{C}= & 2 E_{M}{ }^{C} E^{K}{ }_{[A \mid} \partial_{K} E^{M}{ }_{\mid B]} \\
& +10 d^{M K R} d_{N L R} E_{M}{ }^{C} E^{N}{ }_{B} \partial_{K} E^{L}{ }_{A} .
\end{aligned}
$$

Note that here one does not require the fluxes to be constant as it is done in the generalized Scherk-Shwarz reduction of [46]. The latter generates the scalar potential of the maximal $D=5$ gauged supergravity, where the preservation of supersymmetry requires the so-called linear constraint $\mathcal{F}_{A, B}{ }^{C} \in \mathbf{2 7} \oplus \mathbf{3 5 1}$. In components, one has the trombone $\theta_{A} \in \mathbf{2 7}$ and Z-flux $Z_{A B}{ }^{C} \in \mathbf{3 5 1}[48,49]$.

The components $\mathcal{F}_{A, B}{ }^{C}$ defined in (26) automatically satisfy this constraint, and the corresponding components read

$$
\begin{aligned}
-27 \theta_{A} & =9 \partial_{M} E_{A}^{M}+E_{M}{ }^{B} \partial_{A} E^{M}{ }_{B}, \\
Z_{A B}{ }^{C} & =10 d_{N L R} d^{M K R} E_{M}{ }^{C} E^{N}{ }_{(B \mid} E_{M}{ }^{C} \partial_{K} E^{L}{ }_{\mid A)} .
\end{aligned}
$$

The second line here can be further simplified. Define first the symmetric invariant tensors $d_{A B C}$ and $d^{A B C}$ by explicitly listing the components as in (12) 


$$
\begin{aligned}
d_{b_{1} b_{2}}^{\bar{a} a} & =\frac{1}{\sqrt{5}} \delta_{\left[b_{1}{ }^{\bar{a}} \delta_{b_{2}}\right]^{a},} \\
d_{a_{1} a_{2} a_{3} a_{4} a_{5} a_{6}} & =\frac{1}{4 \sqrt{5}} \epsilon_{a_{1} a_{2} a_{3} a_{4} a_{5} a_{6},}, \\
d_{\bar{a} a} b_{1} b_{2} & =\frac{1}{\sqrt{5}} \delta^{\left[b_{1}\right.} \delta^{\left.b_{a}\right]_{a}}, \\
d^{a_{1} a_{2} a_{3} a_{4} a_{5} a_{6}} & =\frac{1}{4 \sqrt{5}} \epsilon^{a_{1} a_{2} a_{3} a_{4} a_{5} a_{6}} .
\end{aligned}
$$

Due to the nonvanishing weight of the rescaled generalized vielbein $E_{A}^{M} \in E_{6(6)} \times \mathbb{R}^{+}$, the invariant tensors in the curved indices $d^{M N K}$ and $d_{M N K}$ are related to $d_{A B C}$ with flat indices as

$$
d_{M N P} E_{A}^{M} E_{B}^{N} E_{C}^{P}=e^{-3 \phi} e d_{A B C} .
$$

Note that for $\mathcal{E}_{M}{ }^{A}$, one has the expected relation

$$
d_{M N P} \mathcal{E}^{M}{ }_{A} \mathcal{E}^{N}{ }_{B} \mathcal{E}^{P}{ }_{C}=d_{A B C} .
$$

Using this relation, we finally obtain the following expression for Z-flux

$$
Z_{A B}{ }^{C}=5 d_{A B D} d^{M K R} E_{M}^{C}\left(e^{-3 \phi} e \partial_{K} E_{R}^{D}+E_{R}^{D} \partial_{K}\left(e^{-3 \phi} e\right)\right),
$$

which will be used in what follows.

\section{POLYVECTOR DEFORMATIONS}

\section{A. Transformation of fluxes}

In the nonlinear realization approach to the construction of the generalized metric of the exceptional field theory, one finds that the generators of positive level $t^{m_{1} m_{2} m_{3}}$, $t^{m_{1} \ldots m_{6}}$ generate lower triangular generalized vielbein, and the corresponding 3- and 6-forms give the standard field content of supergravity. Working with negative level generators $t_{m_{1} m_{2} m_{3}}, t_{m_{1} \ldots m_{6}}$, one replaces $p$-forms with $p$-vectors generalizing the $\beta$-frame of the double field theory [50] that proves convenient in describing a nongeometric background (see, e.g., [51]). Since both such realizations give a properly defined element of the coset $G / K$, with $G$ and $K$ being the global and local U-duality groups, respectively, it is convenient to define a polyvector deformation as a linear transformation given by the following $\mathrm{E}_{6(6)}$ element

$$
O=\exp \left[\frac{1}{3 !} \Omega^{m n k} t_{m n k}\right] \exp \left[\frac{1}{6 !} \Omega^{m_{1} \ldots m_{6}} t_{m_{1} \ldots m_{6}}\right]
$$

In components, the deformation map $O \in \mathrm{E}_{6(6)}$ can be written as follows

$$
O_{M}^{N}=\left[\begin{array}{ccc}
\delta_{m}{ }^{n} & 0 & 0 \\
-\frac{1}{\sqrt{2}} \Omega^{n m_{1} m_{2}} & \delta^{m_{1}}{ }_{\left[n_{1}\right.} \delta^{m_{2}}{ }_{\left.n_{2}\right]} & 0 \\
\frac{e^{-1}}{2} \delta_{\bar{m}}{ }^{n} J+\frac{e^{-1}}{4} \Omega^{n k l} W_{\bar{m} k l} & -\frac{e^{-1}}{\sqrt{2}} W_{n_{1} n_{2} \bar{m}} & \delta_{\bar{m}}{ }^{\bar{n}}
\end{array}\right],
$$

where we define $J=\frac{e}{6 !} \epsilon_{m_{1} \ldots m_{6}} \Omega^{m_{1} \ldots m_{6}}$ and $W_{m_{1} m_{2} m_{3}}=$ $\frac{e}{3 !} \epsilon_{m_{1} \ldots m_{6}} \Omega^{m_{4} m_{5} m_{6}}$. The barred small Latin indices are the same as the unbarred indices, which are introduced for technical convenience to distinguish between the blocks of the generalized vielbein.

Hence, one defines the deformation of the generalized vielbein and of its inverse as follows

$$
\begin{aligned}
E_{M}{ }^{A} \rightarrow O_{M}{ }^{N} E_{N}{ }^{A} & =\left(\delta_{M}{ }^{N}+\Omega_{M}{ }^{N}\right) E_{N}{ }^{A}, \\
E^{M}{ }_{A} \rightarrow\left(O^{-1}\right)^{M}{ }_{N} E^{N}{ }_{A} & =\left(\delta^{M}{ }_{N}+\tilde{\Omega}^{M}{ }_{N}\right) E^{N}{ }_{A},
\end{aligned}
$$

where one singles out the unity matrix and introduces the covariant deformation tensors $\Omega_{M}{ }^{N}$ and $\tilde{\Omega}^{M}{ }_{N}$. These tensors contain only 3 - and 6-vector deformation parameters, and one naturally decomposes the deformation of the generalized fluxes in powers of $\Omega$ 's. While for $\Omega_{M}{ }^{N}$, the expression is self-evident, for $\widetilde{\Omega}^{M}{ }_{N}$, one obtains

$\tilde{\Omega}_{M}^{N}=\left[\begin{array}{ccc}0 & \frac{1}{\sqrt{2}} \Omega^{n m_{1} m_{2}} & -\frac{e^{-1}}{2} \delta_{\bar{m}}{ }^{n} J+\frac{e^{-1}}{4} \Omega^{n k l} W_{\bar{m} k l} \\ 0 & 0 & \frac{e^{-1}}{\sqrt{2}} W_{n_{1} n_{2} \bar{m}} \\ 0 & 0 & 0\end{array}\right]$.

One now considers the transformation of generalized fluxes under such defined deformations. The simplest option is when generalized fluxes (in flat indices) are invariant upon a condition on the 3 - and 6-index $\rho$-tensors. In the flux formulation of the truncated exceptional field theory this invariance guarantees that the deformation does not spoil the equations of motion, due to all the equations of motion of the scalar sector are written purely in terms of fluxes. For the double field theory, such invariance of generalized fluxes has been observed to be precisely the case when the classical Yang-Baxter equation appears [26]. Moreover, the condition on generalized fluxes in flat indices to transform as scalars appears as a natural condition for fluxes in curved indices to transform covariantly. Indeed, consider

$$
\begin{aligned}
\mathcal{F}^{\prime}{ }_{M N}{ }^{K} & =E_{M}^{\prime}{ }^{A} E_{N}^{\prime}{ }^{B} E_{C}^{\prime}{ }^{K} \mathcal{F}_{A B}^{\prime}{ }^{C} \\
& =O_{M}{ }^{P} O_{N}{ }^{Q}\left(O^{-1}\right){ }^{K}{ }_{L} \mathcal{F}_{P Q}{ }^{L}+\Delta \mathcal{F}_{M N}{ }^{K},
\end{aligned}
$$

where the additional noncovariant term $\Delta \mathcal{F}_{M N}{ }^{K}$ comes from a nontrivial transformation of $\mathcal{F}_{A B}{ }^{C}$. Hence, one concludes that generalized fluxes $\mathcal{F}_{A B}{ }^{C}$ must naturally be scalars under such defined $\mathrm{E}_{6(6)}$ transformations. 
In general, one might be interested in deriving conditions on general $\Omega^{m n k}$ and $\Omega^{m_{1} \ldots m_{6}}$ imposed by the above constraints on transformations of fluxes. However, here we restrict the narrative to only 3 - and 6-Killing deformation, i.e., when

$$
\begin{aligned}
& \Omega^{m_{1} \ldots m_{3}}=\frac{1}{3 !} \rho^{i_{1} \ldots i_{3}} k_{i_{1}}{ }^{m_{1}} \cdots k_{i_{3}}{ }^{m_{3}}, \\
& \Omega^{m_{1} \ldots m_{6}}=\rho^{i_{1} \ldots i_{6}} k_{i_{1}}{ }^{m_{1}} \cdots k_{i_{6}}{ }^{m_{6}} .
\end{aligned}
$$

Here, $\left\{k_{i}{ }^{m}\right\}$ are the Killing vectors of the initial undeformed background, and $\rho^{i_{1} \ldots i_{3}}, \rho^{i_{1} \ldots i_{6}}$ are the constant antisymmetric tensors generalizing the classical $r$-matrix. The Killing vectors satisfy the algebra defined by the usual expression

$$
k_{i_{1}}{ }^{m} \partial_{m} k_{i_{2}}{ }^{n}-k_{i_{2}}{ }^{m} \partial_{m} k_{i_{1}}{ }^{n}=f_{i_{1} i_{2}}{ }^{i_{3}} k_{i_{3}}{ }^{n} .
$$

Consider now the transformation of the trombone flux $\theta_{A}$, for which one finds

$$
\delta \theta_{A}=(\ldots) \rho^{\left[i_{1} \mid i_{2} i_{3}\right.} f_{i_{2} i_{3}}{ }^{\left.\mid i_{4}\right]}+(\ldots) \rho^{\left[i_{1} i_{2} i_{3} i_{4} \mid i_{5} i_{6}\right.} f_{i_{5} i_{6}}{ }^{\left.\mid i_{7}\right]} .
$$

Here, the terms in brackets contain the Killing vectors and various expressions in vielbein and do not contain derivatives of the Killing vectors. Hence, one may naturally impose the following sufficient conditions

$$
\begin{aligned}
\left.\rho^{\left[i_{1} \mid i_{2} i_{3}\right.} f_{i_{2} i_{3}} \mid i_{4}\right] & =0, \\
\left.\rho^{\left[i_{1} i_{2} i_{3} i_{4} \mid i_{5} i_{6}\right.} f_{i_{5} i_{6}} \mid i_{7}\right] & =0 .
\end{aligned}
$$

Recall, for the deformation of the 10d supergravity, one has very similar conditions coming from the trombone flux $r^{i_{1} i_{2}} f_{i_{1} i_{2}}{ }^{i_{3}}=0$, where both lower indices of the structure constants get contracted with the $r$-matrix. This condition guarantees unimodularity of the dual algebra, and being contracted with the Killing vectors, gives the vector $I^{m}$ of generalized supergravity equations of motion. Hence, this unimodularity constraint regulates whether one has a generalized or an ordinary supergravity background upon the deformation, given CYBE is satisfied.

One finds it natural to call the conditions (40) unimodularity constraints, which, indeed, ensures tracelessness of the dual structure constants. One may speculate here on generalized supergravity in 11-dimensions as well as on relaxing this condition, while keeping the flux invariant. Postponing this discussion to Section IV, we notice that the above condition was also found in the analysis of the exceptional Drinfeld algebra and hence, seems the most natural (see below).

While for the invariance of the trombone flux the unimodularity condition is sufficient, the transformation of the flux $Z_{A B}{ }^{C}$ becomes more subtle. Here, one finds it useful to consider the transformation order-by-order in the deformation tensor $\Omega_{M}{ }^{N}$ or equivalently, in $\rho$-tensor, where $\rho^{i_{1} \ldots i_{6}}$ is understood as order two. Hence, one obtains at order one

$$
\left(\delta Z_{A B}{ }^{C}\right)_{1 \mathrm{st}}=(\ldots) \rho^{i_{1} i_{2} i_{3}} f_{i_{2} i_{3}}{ }^{i_{4}},
$$

where, as previously stated, the terms in brackets contain only Killing vectors. Note the absence of antisymmetrization in $\left[i_{1} i_{4}\right]$. From the explicit form of the transformation provided in Appendix B, one concludes that no combination of $\rho$-tensors found in terms of higher order can be used to cancel such first order terms. This forces to strengthen the unimodularity constraint found in the trombone flux and demand

$$
\rho^{i_{1} i_{2} i_{3}} f_{i_{2} i_{3}}{ }^{i_{4}}=0
$$

Note that the condition of precisely this form was found in [35].

At order two, one finds the following

$$
\begin{aligned}
\left(\delta Z_{A B}{ }^{C}\right)_{2 \mathrm{nd}}= & (\ldots) \rho^{\left[i_{1} i_{2} i_{3} i_{4} \mid i_{5} i_{6}\right.} f_{\left.i_{5} i_{6} i_{7}\right]} \\
& +(\ldots)_{\left[i_{2} i_{3} i_{4} i_{7}\right]}\left(\rho^{i_{1} i_{2} i_{6}} \rho^{i_{3} i_{4} i_{5}} f_{i_{5} i_{6}{ }^{i_{7}}}\right. \\
& \left.-\frac{3}{2} \rho^{i_{2} i_{3} i_{4} i_{5} i_{6} i_{7}} f_{i_{5} i_{6}}{ }^{i_{1}}\right)
\end{aligned}
$$

where the terms in the first line reproduce the previously found unimodularity condition for $\rho^{i_{1} \ldots i_{6}}$, and we left the indices $\left[i_{2} i_{3} i_{4} i_{7}\right]$ on the terms in parentheses in the second line to show explicitly the antisymmetry. One finds the terms in brackets projected on the antisymmetric part in $\left[i_{2} i_{3} i_{4} i_{7}\right]$ is the condition sufficient for the second order transformation to vanish. For further comparison with the results of [35], let us rewrite this condition in a more convenient form. Denoting the projection by subscript, we have

$$
\begin{aligned}
& \left(\rho^{i_{1} i_{2} i_{6}} \rho^{i_{3} i_{4} i_{5}} f_{i_{5} i_{6}}^{i_{7}}-\frac{3}{2} \rho^{i_{2} i_{3} i_{4} i_{5} i_{6} i_{7}} f_{i_{5} i_{6}} i_{1}\right)_{\left[i_{2} i_{3} i_{4} i_{7}\right]} \\
& =\left(\rho^{i_{1} i_{2} i_{6}} \rho^{i_{3} i_{4} i_{5}} f_{i_{5} i_{6}}{ }^{i_{7}}-4 f_{i_{5} i_{6}}{ }^{\left[i_{1}\right.} \rho^{\left.i_{2}\right] i_{3} i_{4} i_{5} i_{6} i_{7}}\right)_{\left[i_{2} i_{3} i_{4} i_{7}\right]}
\end{aligned}
$$

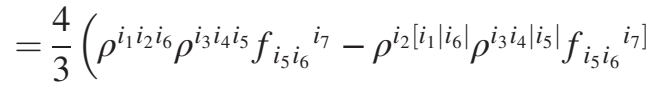

$$
\begin{aligned}
& -3 f_{i_{5} i_{6}}\left[i_{1} \rho^{\left.i_{2}\right] i_{3} i_{4} i_{5} i_{6} i_{7}}\right)_{\left[i_{2} i_{3} i_{4} i_{7}\right]} .
\end{aligned}
$$

Here, in the first line, we used the identity

$5 f_{i_{5} i_{6}}\left[i_{1} \rho^{\left.i_{2} i_{3} i_{4} i_{7}\right] i_{5} i_{6}}+4 f_{i_{5} i_{6}}\left[i_{2} \rho^{i_{1}\left|i_{3} i_{4} i_{7}\right| i_{5} i_{6}}-f_{i_{5} i_{6}}{ }^{i_{1}} \rho^{i_{2} i_{3} i_{4} i_{7} i_{5} i_{6}}=0\right.\right.$ 
and the unimodularity condition on the 6-tensor. In the second line, we used the identity

$$
\begin{aligned}
0 & \equiv f_{i_{5} i_{6}}{ }^{\left[i_{2}\right.} \rho^{i_{1} i_{3}\left|i_{5}\right|} \rho^{\left.i_{4} i_{7}\right] i_{6}} \\
& =\left(f_{i_{5} i_{6}}{ }^{i_{2}} \rho^{i_{1} i_{3} i_{5}} \rho^{i_{4} i_{7} i_{6}}-4 f_{i_{5} i_{6}}\left[i_{1} \mid \rho^{i_{2}\left|i_{3}\right| i_{5}} \rho^{\left.\mid i_{4} i_{7}\right] i_{6}}\right)_{\left[i_{2} i_{3} i_{4} i_{7}\right]},\right.
\end{aligned}
$$

where the most left-hand side (lhs) is due to antisymemtry in $\left[i_{5} i_{6}\right]$, and the right-hand side (rhs) provides a convenient decomposition of the antisymmetrisation $\left[i_{1} i_{2} i_{3} i_{4} i_{7}\right]$. Hence, the sufficient condition that takes into account the symmetries of the remaining terms in the transformation of the generalized flux reads

$$
\begin{aligned}
& \rho^{i_{1}\left[i_{2}\left|i_{6}\right|\right.} \rho^{i_{3} i_{4}\left|i_{5}\right|} f_{\left.i_{5} i_{6}{ }^{i_{7}}\right]}-\rho^{i_{2}\left[i_{1}\left|i_{6}\right|\right.} \rho^{i_{3} i_{4}\left|i_{5}\right|} f_{\left.i_{5} i_{6}{ }^{i_{7}}\right]} \\
& -3 f_{i_{5} i_{6}{ }^{\left[i_{1}\right.} \rho^{\left.i_{2}\right] i_{3} i_{4} i_{5} i_{6} i_{7}}=0 .}=0 .
\end{aligned}
$$

This has precisely the same form as the condition on the $\rho$-tensors obtained in [35] from the analysis of the exceptional Drinfeld algebra; however, up to some additional identifications to be explicitly provided in Sec. III B.

Let us now turn to the analysis of the terms in the transformation of the flux $Z_{A B}{ }^{C}$ of the last third order where one finds an additional constraint

$$
\begin{aligned}
\left(\delta Z_{A B}{ }^{C}\right)_{3 \mathrm{rd}}= & (\ldots)_{\left[i_{3} i_{4} i_{5} i_{6} i_{7} i_{10}\right]} \rho^{i_{1} i_{2}\left[i_{8}\right.} \\
& \times\left(\rho^{i_{3} i_{4} i_{9}} \rho^{\left.i_{5} i_{6} i_{7}\right]} f_{i_{8} i_{9}}{ }^{i_{10}}-18 \rho^{\left.i_{3} i_{4} i_{5} i_{6} i_{7} i_{9}\right]} f_{i_{8} i_{9}}{ }^{i_{10}}\right),
\end{aligned}
$$

where we drop all the terms proportional to the unimodularity and the quadratic constraint (47), and leave explicit indices $\left[i_{3} i_{4} i_{5} i_{6} i_{7} i_{10}\right]$ of the terms in brackets. Hence, one might take the expression cubic in $\rho$-tensor as an additional constraint sufficient for the $\mathrm{E}_{6(6)}$ fluxes to be invariant

$$
\overbrace{\rho^{i_{1} i_{2}\left[i_{8}\right.} \rho^{i_{3} i_{4} i_{9}} \rho^{\left.i_{5} i_{6} i_{7}\right]}}^{\equiv 0} f_{i_{8} i_{9}}{ }^{i_{10}}-18 \rho^{i_{1} i_{2}\left[i_{8}\right.} \rho^{\left.i_{3} i_{4} i_{5} i_{6} i_{7} i_{9}\right]} f_{i_{8} i_{9}}{ }^{i_{10}}=0,
$$

probably with additional antisymmetrization in $\left[i_{3} i_{4} i_{5} i_{6} i_{7} i_{10}\right]$. Notice that the above condition has 7 antisymmetrized indices labeling generators of the symmetry algebra, defined by Killing vectors. This is the reason why such a condition cannot appear in the approach of [35] restricted to group manifolds, when $i, j=1, \ldots, 6$. One expects to find this additional constraint in the $\mathrm{E}_{7(7)}$ exceptional field theory.

Hence, we find the following conditions that are sufficient for the generalized fluxes of the $\mathrm{E}_{6(6)}$ exceptional field theory to be invariant and hence, for a deformation to be solution-generating

$$
\begin{aligned}
& \rho^{i_{1} i_{2} i_{3}} f_{i_{2} i_{3}{ }^{i_{4}}}=0, \\
& \rho^{\left[i_{1} i_{2} i_{3} i_{4} \mid i_{5} i_{6}\right.} f_{i_{5} i_{6}}{ }^{\left.\mid i_{7}\right]}=0, \\
& \rho^{i_{1}\left[i_{2}\left|i_{6}\right|\right.} \rho^{i_{3} i_{4}\left|i_{5}\right|} f_{\left.i_{5} i_{6}{ }^{{ }^{7}}\right]}-\rho^{i_{2}\left[i_{1}\left|i_{6}\right|\right.} \rho^{i_{3} i_{4}\left|i_{5}\right|} f_{i_{5} i_{6}}{ }^{\left.i_{7}\right]} \\
& \quad-3 f_{i_{5} i_{6}}{ }^{\left[i_{1}\right.} \rho^{\left.i_{2}\right] i_{3} i_{4} i_{5} i_{6} i_{7}}=0, \\
& \rho^{i_{1} i_{2}\left[i_{8}\right.} \rho^{\left.i_{3} i_{4} i_{5} i_{6} i_{7} i_{9}\right]} f_{i_{8} i_{9}}{ }^{i_{10}}=0 .
\end{aligned}
$$

First three conditions above are precisely the same as in the EDA approach of [35], whereas the last line is an additional condition, which cannot be seen in the $\mathrm{E}_{6(6)}$ exceptional Drinfeld algebra description of group manifold backgrounds. The equation in the third line above is quadratic in $\rho^{i j k}$ and can be referred to as the generalized YangBaxter equation. Its relation to the construction of exceptional Drinfeld algebras is discussed further in the text. Meaning of the fourth equation as well as its algebraic origin is not as clear.

\section{B. Relation to the algebraic approach of EDA}

The supergravitational analysis above describes the deformation map $O_{M}{ }^{N}$ encoding a 3- and 6-vector deformation of an 11-dimensional background. Using explicit relations between 11-dimensional fields and those of the $\mathrm{E}_{6(6)}$ exceptional field theory, the corresponding generalized metric is defined, whose deformation is a linear transformation. Let us now provide an explicit relation between the $r$-tensors and the constraints on them appearing in the deformation of supergravity backgrounds and those appearing from a constraint on the consistency of the deformations of the $\mathrm{E}_{6(6)}$ exceptional Drinfeld algebra [35].

One first notices that the parametrization of the $\mathbf{2 7}$ employed for the generalized metric here is different from the one used to define the $\mathrm{E}_{6(6)}$ EDA in [35]. Indeed, here, we use $T_{M}=\left\{T_{m}, T^{\left[m_{1}, m_{2}\right]}, T_{\bar{m}}\right\}$, whereas in [35], one has

$$
T_{M}=\left\{T_{m}, T^{\left[m_{1}, m_{2}\right]}, T^{\left[m_{1}, m_{2}, m_{3}, m_{4}, m_{5}\right]}\right\} .
$$

In this form, the origin of the last $\mathbf{6}$ from the windings of the M5-brane is more transparent. Hence, the relation is simply given by the invariant tensor of SL(6)

$$
T_{\bar{m}}=T^{\left[m_{1} m_{2} m_{3} m_{4} m_{5}\right]} \frac{1}{\sqrt{5 !}} \epsilon_{\bar{m}\left[m_{1} m_{2} m_{3} m_{4} m_{5}\right]} .
$$

Next, one compares the deformation map $O_{M}{ }^{N}$ here and the matrix $C_{M}{ }^{N}$ (denoted $C_{A}{ }^{B}$ in the Eq. (4.3) of [35]). While the deformation map $O_{M}{ }^{N}$ acts on the generalized vielbein, and one requires such deformed generalized vielbein to encode a solution, the matrix $C_{M}{ }^{N}$ acts on generators $\left\{T_{M}\right\}$ of the exceptional Drinfeld algebra (EDA), and one requires the transformed generators to form an EDA again. One has 


$$
\begin{aligned}
& O_{M}{ }^{N}=\left[\begin{array}{ccc}
\delta_{m}{ }^{n} & 0 & 0 \\
-\frac{1}{\sqrt{2}} \Omega^{n m_{1} m_{2}} & \delta^{m_{1}}{ }_{n_{1}} \delta^{m_{2}}{ }_{\left.n_{2}\right]} & 0 \\
\frac{e^{-1}}{2} \delta_{\bar{m}}^{n} J+\frac{e^{-1}}{4} \Omega^{n k l} W_{\bar{m} k l} & -\frac{e^{-1}}{\sqrt{2}} W_{n_{1} n_{2} \bar{m}} & \delta_{\bar{m}}{ }^{\bar{n}}
\end{array}\right]
\end{aligned}
$$

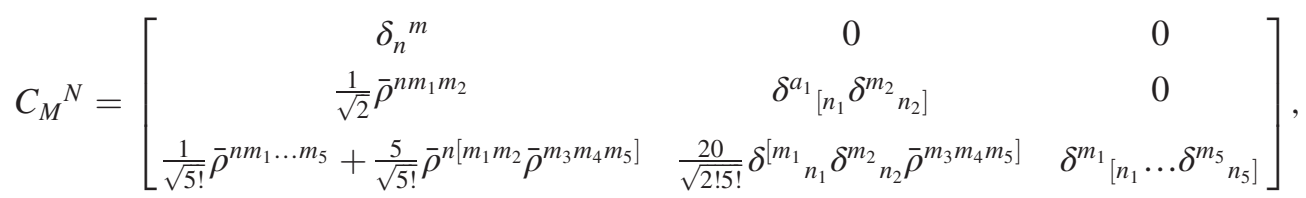

where again $J=\frac{e}{6 !} \epsilon_{m_{1} \ldots m_{6}} \Omega^{m_{1} \ldots m_{6}}$ and $W_{m_{1} m_{2} m_{3}}=$ $\frac{e}{3 !} \epsilon_{m_{1} \ldots m_{6}} \Omega^{m_{4} m_{5} m_{6}}$. For group manifolds, Killing vectors can be identified with the vielbein $e_{i}{ }^{m}=k_{i}{ }^{m}$, and one has

$$
\begin{aligned}
& \Omega^{m_{1} \ldots m_{3}}=\frac{1}{3 !} \rho^{i_{1} i_{2} i_{3}} k_{i_{1}}{ }^{m_{1}} k_{i_{2}}{ }^{m_{2}} k_{i_{3}} m_{3}=\frac{1}{6} \rho^{m_{1} m_{2} m_{3}}, \\
& \Omega^{m_{1} \ldots m_{6}}=\rho^{i_{1} \ldots i_{6}} k_{i_{1}}^{m_{1}} \ldots k_{i_{6}}^{m_{6}}=\rho^{m_{1} \ldots m_{6}},
\end{aligned}
$$

i.e., the $\rho$-tensors now acquire space (curved) indices. Now using $\left[\mu_{1} \ldots \mu_{7}\right]=0$, one rewrites

$$
\delta_{\bar{m}}{ }^{n} J=\delta_{\bar{m}}{ }^{n} \frac{e}{6 !} \epsilon_{n_{1} \ldots n_{6}} \rho^{n_{1} \ldots n_{6}}=\frac{e}{5 !} \epsilon_{\bar{m} n_{1} \ldots n_{5}} \rho^{n n_{1} \ldots n_{5}}
$$

Hence, the deformation map for group manifolds becomes

$$
O_{M}^{N}=\left[\begin{array}{ccc}
\delta_{m}^{n} & 0 & 0 \\
-\frac{1}{6 \sqrt{2}} \rho^{n m_{1} m_{2}} & \left.\delta^{m_{1}}{ }_{n_{1}} \delta^{m_{2}} n_{2}\right] & 0 \\
\frac{1}{5 ! 2} \epsilon_{\bar{m} n_{1} \ldots n_{5}} \rho^{n n_{1} \ldots n_{5}}+\frac{1}{4 \cdot 6^{3}} \rho^{n k l} \epsilon_{\bar{m} k l n_{1} n_{2} n_{3}} \rho^{n_{1} n_{2} n_{3}} & -\frac{1}{36 \sqrt{2}} \epsilon_{m_{1} m_{2} \bar{m} n_{1} n_{2} n_{3}} \rho^{n_{1} n_{2} n_{3}} & \delta_{\bar{m}}{ }^{n}
\end{array}\right]
$$

Comparing this map to the matrix $C_{M}{ }^{N}$ above and using (52), one finds

$$
\begin{aligned}
\rho^{m_{1} m_{2} m_{3}} & =-6 \bar{\rho}^{m_{1} m_{2} m_{3}}, \\
\rho^{m_{1} m_{2} m_{3} m_{4} m_{5} m_{6}} & =2 \bar{\rho}^{m_{1} m_{2} m_{3} m_{4} m_{5} m_{6}} .
\end{aligned}
$$

Now, having at hands the equivalence between transformations encoded by the matrices $O$ and $C$, one may investigate the relations between the constraints on the $\rho$-tensors. The consistency constraints for the generators $T_{M}$ transformed by the matrix $C_{M}{ }^{N}$ to form an EDA derived in [35] read

$$
\begin{aligned}
& \bar{\rho}^{m_{1} m_{2} m_{3}} f_{m_{1} m_{2}}^{m_{4}}=0, \quad \text { unimodularity } 3, \\
& \left.\bar{\rho}^{m_{1} m_{2}\left[m_{3} \ldots m_{6}\right.} f_{m_{1} m_{2}} m_{7}\right]=0, \quad \text { unimodularity } 6 \text {, }
\end{aligned}
$$

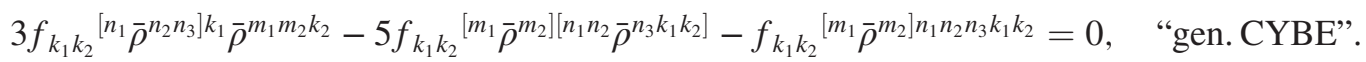

While the unimodularity conditions in the first two lines are precisely the same as the ones following from the flux invariance, the third line requires more work. Let us expand the antisymmetrization in $\left[n_{1} n_{2} n_{3} k_{1} k_{2}\right]$ of the third line above and reorganize it in the following more symmetric form

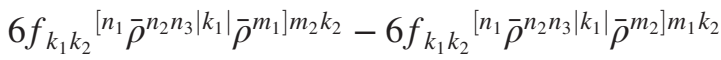

$$
\begin{aligned}
& =f_{k_{1} k_{2}}{ }^{\left[m_{1}\right.} \bar{\rho}^{\left.m_{2}\right] n_{1} n_{2} n_{3} k_{1} k_{2}} .
\end{aligned}
$$

Upon (57) this becomes precisely (47).

\section{The short SL(5) story}

Let us now briefly look at 3-vector deformations in the formalism of the SL(5) exceptional field theory and show that the only condition appearing both in the algebraic and ExFT approaches is the unimodularity constraint.

Here, we work in the same conventions as those in [32] and for the sake of brevity, we will avoid a lengthy description of the SL(5)-covariant exceptional field theory. For the details of the construction of the SL(5) exceptional field theory, see [52,53]. However, it is important to mention the generalized Lie derivative of the generalized vielbein 


$$
\begin{aligned}
\mathcal{L}_{\Lambda} E^{M}{ }_{A}= & \frac{1}{2} \Lambda^{K L} \partial_{K L} E^{M}{ }_{A}+\partial_{K L} \Lambda^{M K} E^{L}{ }_{A} \\
& +\frac{1}{4} \partial_{K L} \Lambda^{K L} E^{M}{ }_{A},
\end{aligned}
$$

whose explicit form reflects the fact that we work in the truncated theory. Here, $M, N, K, \ldots=1, \ldots, 5$ label the coordinate indices, whereas $A, B, C, \ldots=1 \ldots 5$ label the flat indices. As previously accepted, the small Latin indices $m, n, k, \ldots$ and $a, b, c, \ldots$ label the directions of the "internal" space, which is four-dimensional in this case.

Explicitly, the generalized metric and the deformation map are given by

$$
\begin{gathered}
E_{M}{ }^{A}=e^{\frac{\phi}{2}}\left[\begin{array}{cc}
e^{-1 / 2} e_{m}{ }^{a} & e^{1 / 2} v^{a} \\
0 & e^{1 / 2}
\end{array}\right], \\
O_{M}{ }^{N}=\left[\begin{array}{cc}
\delta_{m}{ }^{n} & 0 \\
\frac{1}{3 !} \epsilon_{m p q r} \Omega^{p q r} & 1
\end{array}\right],
\end{gathered}
$$

where $v^{m}=\frac{e^{-1}}{3 !} \epsilon^{m n k l} C_{n k l}$ and, as before, $\Omega^{m_{1} m_{2} m_{3}}=$ $\frac{1}{3 !} \rho^{i_{1} i_{2} i_{3}} k_{i_{1}}{ }^{m_{1}} k_{i_{2}}{ }^{{ }_{2}} k_{i_{3}}{ }^{m_{3}}$. The generalized flux is defined as structure constants of the corresponding Leibniz algebra, as follows:

$$
\mathcal{L}_{E_{A B}} E^{M}{ }_{C}=\mathcal{F}_{A B, C}{ }^{D} E^{M}{ }_{D},
$$

where $E_{A B}{ }^{M N}=2 E_{\left[A^{M}\right.} E_{B]}{ }^{N}$. In [54], it was shown explicitly that such defined flux contains only the 10,15 , and $\overline{\mathbf{4 0}}$ of SL(5), which in tensor notations are encoded by the trombone $\theta_{[A B]}$ and by fluxes $Y_{(A B)}$ and $Z^{A B, C}$. Explicitly, one defines the irreducible flux components as $Y_{(B C)}=$ $\mathcal{F}_{A(B, C)}{ }^{A}, \quad \theta_{[B C]}=\mathcal{F}_{A[B, C]}{ }^{A}, \quad \mathcal{F}_{[A B C]}{ }^{D}=Z_{A B C}{ }^{D}+\theta_{[A B} \delta_{C]}^{D}$, which gives

$$
\begin{aligned}
\theta_{A B}= & E^{-1} E^{M N}{ }_{A B} \partial_{M N} E-E^{M}{ }_{[A} \partial_{M N} E^{N}{ }_{B]}, \\
Y_{A B}= & E^{M}{ }_{(A} \partial_{M N} E^{N}{ }_{B)}, \\
Z_{A B C}{ }^{D}= & E_{[A}^{M} E_{B \mid}^{N} E_{K}^{D} \partial_{M N} E_{\mid C]}^{K}+\frac{1}{3}\left(2 E_{[A \mid}^{M} \partial_{M N} E_{|B|}^{N}\right. \\
& \left.+E_{[A}^{M} E_{B \mid}^{N} E^{-1} \partial_{M N} E\right) \delta_{\mid C]}^{D} .
\end{aligned}
$$

Here, we denote $E=\operatorname{det} E^{A}{ }_{M}$. Following the same procedure as for the $\mathrm{E}_{6(6)}$ case, one finds that the transformation of all components of the generalized flux at all orders in $\rho^{i_{1} i_{2} i_{3}}$ can be written as

$$
\delta \mathcal{F}_{A B, C}{ }^{D}=(\ldots) \rho^{i_{1} i_{2} i_{3}} f_{i_{2} i_{3}}{ }^{i_{4}} .
$$

Hence, the unimodularity constraint is, indeed, sufficient for the flux to be invariant and for the deformation to generate a solution.

At the algebraic side, one considers the SL(5) exceptional Drinfeld algebra developed in $[10,11]$ and deforms generators, as follows:

$$
\begin{aligned}
T_{m} & \rightarrow T_{m}, \\
T^{m n} & \rightarrow T^{m n}+\rho^{m n k} T_{k} .
\end{aligned}
$$

Requiring the deformed generators to also form an SL(5) exceptional Drinfeld algebra, one derives the following constraints (see [10] for more detail)

$$
\begin{aligned}
\rho^{m_{1} m_{2} m_{3}} f_{m_{2} m_{3}{ }^{m_{4}}}=0, \\
4 f_{k_{1} k_{2}}{ }^{\left[m_{1}\right.} \rho^{\left.m_{2}\right] k_{1}\left[n_{1}\right.} \rho^{\left.n_{2} n_{3}\right] k_{2}}+3 f_{k_{1} k_{2}{ }^{\left[n_{1}\right.}} \rho^{\left.n_{2} n_{3}\right] k_{1}} \rho^{m_{1} m_{2} k_{2}}=0 .
\end{aligned}
$$

The first line is simply the unimodularity constraint whose appearance was expected. The second line is a quadratic constraint, which is, however, equivalent to the first line. Indeed, since the indices $m, n, k=1, \ldots 4$, it is natural to define

$$
\rho_{m}=\epsilon_{m n k l} \rho^{n k l} .
$$

Substituting this into the second line of (67) and contracting the indices $m_{1} m_{2}$ and $n_{1} n_{2}$ with epsilon-tensor, one rewrites the constraint as

$$
\rho_{m_{1}} \rho_{\left[n_{1}\right.} f_{\left.n_{2} n_{3}\right]^{m_{1}}}=0
$$

which vanishes identically upon the unimodularity constraint. Note that while one is able to define such $\rho_{m}$ only on group manifold, we see that the unimodularity constraint is still sufficient for a tri-vector SL(5) deformation to generate solutions.

One notices, however, that the non-Abelian deformation provided in [34] are all nonunimodular, but still provide solutions. This perfectly illustrates the fact that such obtained conditions are only sufficient rather than necessary. At the same time, this observation again raises the question of searching for nontrivial tri-vector deformations, now satisfying the generalized classical YangBaxter equation.

\section{CONCLUSIONS AND DISCUSSIONS}

In this work, we investigate 3- and 6-Killing deformations of general backgrounds of the 11-dimensional supergravity admitting at least three Killing vectors, not necessarily commuting. The formalism of the exceptional field theory provides more natural degrees of freedom for 
the deformations than the conventional formulation of supergravity. In these terms, tri-vector deformations appear to be encoded by an $\mathrm{E}_{d(d)}$ element generated by a 3- and 6-vector. Since the field content of ExFT is given by tensor of GL(11-d) taking values in irreps of the U-duality group, these transform linearly. Schematically that means

$\Phi_{\mu_{1} \ldots \mu_{p}}{ }^{M_{1} \ldots M_{N}} \rightarrow O_{N_{1}}{ }^{M_{1}} \ldots O_{N_{N}}{ }^{M_{N}} \Phi_{\mu_{1} \ldots \mu_{p}}{ }^{N_{1} \ldots N_{N}}$.

Here, the indices $\mu=1 \ldots 11-d$, the indices $M, N$ label certain irrep of $\mathrm{E}_{d(d)}$ and the matrix $O$ is defined as in (32), and we restrict the polyvectors to be proportional to Killing vectors of the initial background

$$
\begin{aligned}
& \Omega^{m_{1} \ldots m_{3}}=\frac{1}{3 !} \rho^{i_{1} \ldots i_{3}} k_{i_{1}}{ }^{m_{1}} \cdots k_{i_{3}}{ }^{m_{3}}, \\
& \Omega^{m_{1} \ldots m_{6}}=\rho^{i_{1} \ldots i_{6}} k_{i_{1}}{ }^{m_{1}} \cdots k_{i_{6}}{ }^{m_{6}} .
\end{aligned}
$$

Working for concreteness in the $\mathrm{E}_{6(6)}$ exceptional field theory, we consider its truncation to the scalar sector as in [32]. This leaves us with only the generalized metric parametrizing the coset $\mathrm{E}_{6(6)} \times \mathbb{R}^{+} / \mathrm{Usp}(8)$. Explicitly, the corresponding generalized vielbein is given in (20). While the generalized vielbein transforms under the deformations linearly, the transformation of the supergravity fields entering its definition are nonobvious. The covariant formalism allows us to refrain from attempting to provide explicit formulas for the deformations of supergravity fields, as it was done in [32]. Such analysis, however, would be necessary for one to generate explicit examples of deformations within the $\mathrm{E}_{6(6)}$ theory.

Truncated exceptional field theory can be completely written in terms of generalized fluxes, as it was explicitly shown in [46] for the $E_{6(6)}$ symmetry group. The corresponding equations of motion for the generalized vielbein can as well be written in terms of fluxes with a single factor of the inverse vielbein $E_{A}{ }^{M}$. Hence, the sufficient condition for the deformation to map a solution of such equations of motion to a solution is the condition for generalized fluxes to transform covariantly. Hence, fluxes with local $\mathrm{USp}(8)$ indices $\mathcal{F}_{A B}{ }^{C}$ must be invariant. We investigate the transformation of generalized fluxes under such defined transformations and find out that one may ensure the invariance imposing conditions on the constant tensors $\rho^{i_{1} i_{2} i_{3}}$ and $\rho^{i_{1} \ldots i_{6}}$. These tensors are the generalization of the classical $r$-matrix, and the condition is believed to be the generalization of the classical Yang-Baxter equation. We show that the condition sufficient for the deformation to be a solution-generating transformation is precisely derived via the $\rho$-deformation of the $\mathrm{E}_{6(6)}$ exceptional Drinfeld algebra in [11].
The same analysis of generalized fluxes of the truncated SL(5) theory shows that the sufficient condition is simply the unimodularity constraint on $\rho^{i_{1} i_{2} i_{3}}$, i.e.,

$$
\rho^{i_{1} i_{2} i_{3}} f_{i_{2} i_{3}}{ }^{i_{4}}=0
$$

We notice that the quadratic constraint on $\rho^{i_{1} i_{2} i_{3}}$ derived in [10] is satisfied identically upon the unimodularity constraint, which provides consistency between supergravity and the EDA picture.

It is worth here to return back to the result of [32], where explicit examples of tri-vector deformations of $\mathrm{AdS}_{4} \times \mathbb{S}^{7}$ based on the SL(5) approach were presented. One checks that all non-Abelian examples there are nonunimodular, which naively seems to contradict the above result. However, since the lhs of the unimodularity condition gets contracted with subtle expressions in vielbeins, 3-forms and Killing vectors, the condition is far from being necessary. One concludes that the results of [32] provide examples of tri-Killing deformations that are not (generalized) YangBaxter in the above sense. To our knowledge, the literature does not contain examples of such non-Yang-Baxter bivector deformations relevant for two-dimensional sigmamodels. It would be interesting to search for examples of such deformations in more space-time dimensions, where one has nontrivial CYBE in addition to the unimodularity constraint.

Given the explicit check of the invariance of (internal) generalized fluxes of the $E_{6(6)}$ exceptional field theory, where at the end of the day subtle and long terms boil down to relatively simple expressions containing the algebraic equation, one might expect the same to happen for all fields of any exceptional field theory. Since, as we discussed above, the examples of tri-vector deformations of [32] stand slightly apart from the standard narrative of YangBaxter deformations, it is still an interesting problem to find examples of deformation that do satisfy the generalized Yang-Baxter equation (50). Another interesting direction for further research is to investigate tri-vector deformations of the 10-dimensional supergravity and in particular, twodimensional sigma-models, which raises intriguing questions of integrability of such deformations. This is work in progress, and results will be reported in further publications.

\section{ACKNOWLEDGMENTS}

This work was supported by Russian Science Foundation Grant No. RSCF-20-72-10144. The authors acknowledge discussions with I. Bakhmatov, E. Malek, N.S. Deger, who motivated this project, and thank E. M. Bazanova for useful comments on the text. 


\section{APPENDIX A: NOTATIONS AND CONVENTIONS}

In this paper, we use the following conventions for indices

$$
\begin{aligned}
& \hat{\mu}, \hat{\nu},=1 \ldots 11 \quad \text { eleven directions, curved; } \\
& \hat{\alpha}, \hat{\beta},=1 \ldots 11 \quad \text { eleven directions, flat; } \\
& \mu, \nu, \rho, \ldots=1 \ldots 5 \quad \text { external directions of ExFT, curved; } \\
& \bar{\mu}, \bar{\nu}, \bar{\rho}, \ldots=1 \ldots 5 \quad \text { external directions of ExFT, flat; } \\
& k, l, m, n, \ldots=1, \ldots, 6 \quad \text { internal six directions, curved; } \\
& \bar{k}, \bar{l}, \bar{m}, \bar{n}, \ldots=1, \ldots, 6 \quad \text { dual internal six directions, curved; } \\
& a, b, c, d, \ldots=1, \ldots, 6 \quad \text { internal six directions, flat; } \\
& \bar{a}, \bar{b}, \bar{c}, \bar{d}, \ldots=1, \ldots, 6 \quad \text { dual internal six directions, flat; } \\
& M, N, K, L, \ldots=1, \ldots, 27 \quad \text { fundamental ExFT indices, curved; } \\
& A, B, C, D, \ldots=1, \ldots, 27 \quad \text { fundamental ExFT indices, flat; } \\
& i, j=1, \ldots, N \quad \text { indices labeling Killing vectors; }
\end{aligned}
$$

\section{APPENDIX B: DERIVATION OF FLUX TRANSFORMATIONS}

Tedious part of calculations involved with explicit substitution of the deformed vielbein into flux expressions, decomposition, and preliminary simplification of the result were done using the computer algebra program Cadabra v.1.4 [55,56] (note that v.2 is also available [57]). The corresponding Cadabra files can be found here [58].

One starts with the trombone flux whose transformation becomes

$$
\begin{aligned}
& \delta \theta_{A}=-3 \frac{1}{\sqrt{2}} E_{m n A} k_{i_{1}}{ }^{m} \rho^{\left[i_{1} \mid i_{2} i_{3}\right.} f_{i_{2} i_{3}}{ }^{\left.\mid i_{4}\right]} k_{i_{4}}{ }^{n}+\frac{1}{96} E^{\bar{k}}{ }_{A} \epsilon_{\bar{k} l m n p q} k_{i_{1}}{ }^{l} k_{i_{2}}{ }^{m} k_{i_{3}}{ }^{n} k_{i_{4}}{ }^{p} \rho^{i_{1} i_{2} i_{3}} \rho^{\left[i_{4} \mid i_{5} i_{6}\right.} f_{i_{5} i_{6}}{ }^{\left.\mid i_{7}\right]} k_{i_{7}}{ }^{q} \\
& -\frac{3}{16} E^{\bar{k}}{ }_{A} \epsilon_{\bar{k} l m n p q} k_{i_{1}}{ }^{l} k_{i_{2}}{ }^{m} k_{i_{3}}{ }^{n} k_{i_{4}}{ }^{p} \rho^{\left[i_{1} i_{2} i_{3} i_{4} \mid i_{5} i_{6}\right.} f_{i_{5} i_{6}}{ }^{\left.\mid i_{7}\right]} k_{i_{7}}{ }^{q} \\
& +\frac{1}{96} E^{\bar{k}}{ }_{A} \epsilon_{\bar{k} l m n p q} k_{i_{1}}{ }^{l}{k_{i_{2}}}^{m} k_{i_{3}}{ }^{n}{k_{i_{4}}}^{p} \rho^{i_{1} i_{2} i_{3}} \rho^{i_{5} i_{6}\left[i_{7} \mid\right.} f_{i_{5} i_{6}}{ }^{\left.\mid i_{4}\right]} k_{i_{7}}{ }^{q} \\
& \sim(\ldots) \underbrace{\left.\rho^{\left[i_{1} \mid i_{2} i_{3}\right.} f_{i_{2} i_{3}} i_{4}\right]}_{\text {unimodularity }}+(\ldots) \underbrace{\rho^{\left[i_{1} i_{2} i_{3} i_{4} \mid i_{5} i_{6}\right.} f_{i_{5} i_{6}}{ }^{\left.\mid i_{7}\right]}}_{\text {unimodularity } 6} .
\end{aligned}
$$

One observes that terms of both first and second order in the $\rho$-tensors are proportional to the unimodularity constraint. Note that although here the constraint comes with antisymmetrization of the upper indices, further analysis of the Z-flux will require a more strict version of the constraint without antisymmetrization

$$
\rho^{i_{2} i_{3} i_{4}} f_{i_{2} i_{3}}^{i_{1}}=0
$$

The transformation of the Z-flux is convenient to analyze order-by-order in the $\rho$-tensor assuming that $\rho^{i_{1} i_{2} i_{3}}$ is of order one, while $\rho^{i_{1} \ldots i_{6}}$ is of order two. Hence, at first order, one has

$$
\begin{aligned}
\left(\delta Z_{A B}{ }^{C}\right)_{1 \mathrm{st}}= & \frac{5 e}{6 \sqrt{10}} d_{A B D}\left(E_{\bar{m}}{ }^{C} E_{n}{ }^{D} k_{i_{1}}{ }^{\bar{m}} \rho^{i_{2} i_{3}\left[i_{4} \mid\right.} f_{i_{2} i_{3}}{ }^{\left.\mid i_{1}\right]} k_{i_{4}}{ }^{n}+E_{\bar{m}}{ }^{[D} E_{n}{ }^{C]} k_{i_{1}}{ }^{\bar{m}} \rho^{i_{2} i_{3} i_{4}} f_{i_{2} i_{3}}{ }^{i_{1}} k_{i_{4}}{ }^{n}\right. \\
& \left.+\frac{1}{4} E^{k l C} E^{m n D} \epsilon_{k l m n p q} k_{i_{1}}{ }^{p} \rho^{i_{2} i_{3}\left[i_{1}\right.} f_{i_{2} i_{3}}{ }^{\left.i_{4}\right]} k_{i_{4}}{ }^{q}\right)
\end{aligned}
$$

which again vanishes upon the unimodularity constraint. 
Next, at order two, one writes

$$
\begin{aligned}
& \left(\delta Z_{A B}{ }^{C}\right)_{2 \text { nd }}=\frac{e}{24 \sqrt{5}} d_{A B D} \epsilon_{\text {lmnpqr }}\left(\frac{1}{12} E_{k}{ }^{(D \mid} E^{l m \mid C)} i_{1}{ }^{k}{ }_{i_{2}}{ }^{n}{ }_{i_{3}}{ }^{p}{ }_{i_{4}}{ }^{q} \rho^{i_{1}\left[i_{2} i_{3} \mid\right.} \rho^{i_{5} i_{6} \mid i_{7}} f_{i_{5} i_{6}}{ }^{\left.i_{4}\right]}\right. \\
& +\frac{1}{18} E_{k}{ }^{D} E^{l m C} K_{i_{1}}{ }^{k} i_{2}{ }^{n} i_{3}{ }^{p}{ }^{p}{ }^{q}{ }^{q} \rho^{i_{2} i_{3} i_{4}} \rho^{i_{5} i_{6}\left[i_{1}\right.} f_{i_{5} i_{6}}{ }^{\left.i_{7}\right]}+\frac{1}{36} E_{k}{ }^{[D \mid} E^{l m \mid C]} K_{i_{1}}{ }^{k}{ }_{i_{5}}{ }^{p}{ }_{i_{6}}{ }^{q}{ }_{i_{4}}{ }^{n} \rho^{i_{5} i_{6} i_{7}} \rho^{i_{1} i_{2} i_{3}} f_{i_{2} i_{3}}{ }^{i_{4}} \\
& +\frac{1}{6} E_{k}{ }^{[D \mid} E^{l m \mid C]} K_{i_{1}}{ }^{k}{ }_{i_{2}}{ }^{n}{ }_{i_{3}}{ }^{p}{ }_{i_{4}}{ }^{q} \rho^{i_{1}\left[i_{2} \mid i_{6}\right.} \rho^{\left|i_{3} i_{4}\right| i_{5}} f_{i_{5} i_{6}}{ }^{\left.\mid i_{7}\right]}-\frac{1}{4} E_{k}{ }^{D} E^{l m C} K_{i_{1}}{ }^{k}{ }_{i_{2}}{ }^{n}{ }_{i_{3}}{ }^{p}{ }_{i_{4}}{ }^{q} \rho^{i_{2} i_{3} i_{4} i_{5} i_{6} i_{7}} f_{i_{5} i_{6}}{ }^{i_{1}} \\
& \left.-E_{k}{ }^{(D \mid} E^{l m \mid C)} K_{i_{1}}{ }^{k} i_{2}{ }^{n} i_{3}{ }^{p}{ }_{i_{4}}{ }^{q} \rho^{i_{1}\left[i_{2} i_{3} i_{4} \mid i_{5} i_{6}\right.} f_{i_{5} i_{6}}{ }^{\left.\mid i_{7}\right]}\right) i_{7}{ }^{r},
\end{aligned}
$$

where, for clarity of expressions, we define

$$
K_{i_{1} \ldots i_{p}}^{m_{1} \ldots m_{p}}=k_{i_{1}}^{{ }^{m_{1}}} \ldots k_{i_{p}}{ }^{m_{p}}
$$

Given the unimodularity constraint, the first three lines vanish, and the above expression becomes

$$
\begin{aligned}
& \left(\delta Z_{A B}{ }^{C}\right)_{2 \text { nd }}=\frac{e}{24 \sqrt{5}} d_{A B D} \epsilon_{l m n p q r}\left(\frac{1}{6} E_{k}{ }^{[D \mid} E^{I m \mid C]} K_{i_{1}}{ }^{k}{ }_{i_{2}}{ }^{n}{ }_{i_{3}}{ }^{p}{ }_{i_{4}}{ }^{q} \rho^{i_{1}\left[i_{2} \mid i_{6}\right.} \rho^{\left|i_{3} i_{4}\right| i_{5}} f_{i_{5} i_{6}}{ }^{\left.\mid i_{7}\right]}\right. \\
& \left.-\frac{1}{4} E_{k}{ }^{D} E^{l m C} K_{i_{1}}{ }^{k}{ }_{i_{2}}{ }^{n}{ }_{i_{3}}{ }^{p}{ }_{i_{4}}{ }^{q} \rho^{i_{2} i_{3} i_{4} i_{5} i_{6} i_{7}} f_{i_{5} i_{6}}{ }^{i_{1}}-E_{k}{ }^{(D \mid} E^{l m \mid C)} K_{i_{1}}{ }^{k}{ }_{i_{2}}{ }^{n}{ }_{i_{3}}{ }^{p}{ }_{i_{4}}{ }^{q} \rho^{i_{1}\left[i_{2} i_{3} i_{4} \mid i_{5} i_{6}\right.} f_{i_{5} i_{6}}{ }^{\left.\mid i_{7}\right]}\right)_{i_{7}}{ }^{r} .
\end{aligned}
$$

Using the identity

$$
\rho^{i_{1}\left[i_{2} i_{3} i_{4} \mid i_{5} i_{6}\right.} f_{i_{5} i_{6}}{ }^{\left.\mid i_{7}\right]}=\frac{5}{4} \rho^{\left[i_{1} i_{2} i_{3} i_{4} \mid i_{5} i_{6}\right.} f_{i_{5} i_{6}}{ }^{\left.\mid i_{7}\right]}-\frac{1}{4} \rho^{i_{2} i_{3} i_{4} i_{7} i_{5} i_{6}} f_{i_{5} i_{6}}{ }^{i_{1}},
$$

and performing a series of algebraic manipulations, the final expression can be "massaged" to

$$
\begin{aligned}
& \left(\delta Z_{A B}{ }^{C}\right)_{2 \text { nd }}=-\frac{5 \sqrt{5} e}{96} d_{A B D} E_{k}{ }^{(D \mid} E^{l m \mid C)} \epsilon_{l m n p q r} K_{i_{1}}{ }^{k} i_{2}{ }^{n}{ }^{p}{ }^{p}{ }^{p}{ }_{i_{4}}{ }^{q}{ }^{r}{ }^{r}{ }^{r} \rho^{\left[i_{1} i_{2} i_{3} i_{4} \mid i_{5} i_{6}\right.} f_{i_{5} i_{6}}{ }^{\left.\mid i_{7}\right]} \\
& +\frac{\sqrt{5} e}{3 \cdot 48} d_{A B D} E_{k}{ }^{[D \mid} E^{l m \mid C]} \epsilon_{\text {lmnpqr }} K_{i_{1}}{ }^{k}{ }_{i_{2}}{ }^{n}{ }_{i_{3}}{ }^{p}{ }_{i_{4}}{ }^{q}{ }_{i_{7}}{ }^{r}\left(\rho^{i_{1}\left[i_{2} \mid i_{6}\right.} \rho^{\left|i_{3} i_{4}\right| i_{5}} f_{i_{5} i_{6}}{ }^{\left.\mid i_{7}\right]}-\frac{3}{2} \rho^{\left(i_{2} i_{3} i_{4} i_{5} i_{6} i_{7}\right.} f_{i_{5} i_{6}}{ }^{\left.i_{1}\right)}\right) .
\end{aligned}
$$

The yellow expression in the first line is the unimodularity condition for the tensor $\rho^{i_{1} \ldots i_{6}}$, whereas the black terms in the second line compose the generalized classical Yang-Baxter equation. The latter is equivalent to the constraint obtained in [35].

Finally, the terms of third order in the $\rho$-tensors read

$$
\begin{aligned}
& \left(\delta Z_{A B}{ }^{C}\right)_{3 \mathrm{~d}}=\frac{e}{96 \sqrt{10}} d_{A B D}\left(\frac{5}{54} E_{k}{ }^{C} E_{l}{ }^{D} \epsilon_{m n p q r t} K_{i_{1}}{ }^{k}{ }_{i_{10}}{ }^{l}{ }_{i_{2}}{ }^{m}{ }_{i_{3}}{ }^{n}{ }_{i_{4}}{ }^{p}{ }_{i_{5}}{ }^{q}{ }_{i_{6}}{ }^{r} \rho^{i_{1} i_{2} i_{3}} \rho^{i_{4} i_{5} i_{6}} \rho^{i_{7} i_{8} i_{9}} f_{i_{7} i_{8}}{ }^{i_{10}}{ }_{i_{9}}{ }^{t}\right. \\
& +\frac{5}{54} E_{k}{ }^{(C} E_{l}{ }^{D)} \epsilon_{\text {mnpqrt }} K_{i_{1}}{ }^{k}{ }_{i_{10}}{ }^{l}{ }_{i_{2}}{ }^{m}{ }_{i_{3}}{ }^{n}{ }_{i_{4}}{ }^{p}{ }_{i_{5}}{ }^{q}{ }_{i_{6}}{ }^{r}{ }_{i_{9}}{ }^{t} \rho^{i_{1} i_{2} i_{3}} \rho^{i_{5} i_{6} i_{9}} \rho^{i_{10} i_{7} i_{8}} f_{i_{7} i_{8}{ }^{i_{4}}} \\
& +\frac{1}{18} E_{k}{ }^{C} E_{l}{ }^{D} \epsilon_{\text {mnpqrt }} K_{i_{1}}{ }^{k} i_{10}{ }^{l}{ }_{i_{2}}{ }^{m}{ }_{i_{3}}{ }^{n}{ }_{i_{4}}{ }^{p}{ }_{i_{5}}{ }^{q}{ }_{i_{6}}{ }^{r}{ }_{i_{9}}{ }^{t} \rho^{i_{2} i_{3} i_{4} i_{5} i_{6} i_{9}} \rho^{i_{7} i_{8}\left[i_{10}\right.} f_{\left.i_{7} i_{8}{ }^{i_{1}}\right]} \\
& -5 \sqrt{2} E_{k}{ }^{D} E^{l m C} \epsilon_{\text {lmnpqr }} K_{i_{1}}{ }^{k}{ }_{i_{2}}{ }^{n}{ }_{i_{3}}{ }^{p}{ }_{i_{4}}{ }^{q}{ }_{i_{7}}{ }^{r} \rho^{i_{2} i_{3} i_{4} i_{5} i_{6} i_{7}} f_{i_{5} i_{6}{ }^{i_{1}}}
\end{aligned}
$$




$$
\begin{aligned}
& -4 \sqrt{2} E_{k}{ }^{(D \mid} E^{l m \mid C)} \epsilon_{\text {lmnpqr }} K_{i_{1}}{ }^{k} i_{2}{ }^{n} i_{3}{ }^{p}{ }_{i_{4}}{ }^{q} \rho^{i_{1}\left[i_{2} i_{3} i_{4} \mid i_{5} i_{6}\right.} f_{i_{5} i_{6}}{ }^{\left|i_{7}\right|}{ }_{i_{7}}{ }^{r} \\
& +\frac{5}{27} E_{k}{ }^{C} E_{l}{ }^{D} \epsilon_{\text {mnpqrt }} K_{i_{1}}{ }^{k}{ }_{i_{2}}{ }^{l} i_{3}{ }^{m}{ }_{i_{4}}{ }^{n}{ }_{i_{5}}{ }^{p}{ }_{i_{6}}{ }^{q}{ }_{i_{7}}{ }^{r} i_{10}{ }^{t} \rho^{i_{1}\left[i_{3} \mid i_{8}\right.} \rho^{i_{2}\left|i_{4}\right| i_{9}} \rho^{\mid i_{5} i_{6} i_{7}} f_{i_{8} i_{9}} e^{\left.i_{10}\right]} \\
& -\frac{5}{54} E_{k}{ }^{C} E_{l}{ }^{D} \epsilon_{m n p q r t} K_{i_{1}}{ }^{k} i_{2}{ }^{l} i_{3}{ }^{m}{ }_{i_{4}}{ }^{n}{ }^{n}{ }_{5}{ }^{p}{ }_{i_{6}}{ }^{q}{ }_{i_{7}}{ }^{r} i_{10}{ }^{t} \rho^{i_{1}\left[i_{3} \mid i_{8}\right.} \rho^{\left|i_{10} i_{4}\right| i_{9}} \rho^{\left.\mid i_{5} i_{6} i_{7}\right]} f_{i_{8} i_{9} e^{i_{2}}} \\
& +\frac{5}{18} E_{k}{ }^{C} E_{l}{ }^{D} \epsilon_{\text {mnpqrt }} K_{i_{1}}{ }^{k} i_{2}{ }^{l} i_{3}{ }^{m}{ }_{i_{4}}{ }^{n}{ }_{i_{5}}{ }^{p}{ }_{i_{6}}{ }^{q}{ }_{i_{7}}{ }^{r}{ }_{i_{10}}{ }^{t} \rho^{i_{1}\left[i_{3} \mid i_{8}\right.} \rho^{\left|i_{7} i_{4}\right| i_{9}} \rho^{\left|i_{5} i_{6}\right| i_{2}} f_{i_{8} i_{9} e^{\left.\mid i_{10}\right]}} \\
& \left.+\frac{1}{3} E_{k}{ }^{C} E_{l}{ }^{D} \epsilon_{m n p q r t} K_{i_{1}}{ }^{k}{ }_{i_{2}}{ }^{l} i_{3}{ }^{m} i_{i_{4}}{ }^{n} i_{5}{ }^{p}{ }_{i_{6}}{ }^{q}{ }_{i_{7}}{ }^{r}{ }_{i_{10}}{ }^{t} \rho^{i_{1} i_{2} i_{9}} \rho^{\left[i_{3} i_{4} i_{5} i_{6} i_{7} \mid i_{8}\right.} f_{i_{8} i_{9}} e^{\left.\mid i_{10}\right]}\right) \text {. }
\end{aligned}
$$

The first five lines vanish because of the unimodularity constraint, whereas the rest can be neatly packaged, as follows:

$$
\begin{aligned}
& \left(\delta Z_{A B}{ }^{C}\right)_{3 \mathrm{rd}}=\frac{\sqrt{5} e}{5184 \sqrt{2}} d_{A B D} E_{k}{ }^{C} E_{l}{ }^{D} \epsilon_{\text {mnpqrt }} K_{i_{1}}{ }^{k} i_{2}{ }^{l} i_{3}{ }^{m}{ }_{i_{4}}{ }^{n}{ }_{i_{5}}{ }^{p}{ }_{i_{6}}{ }^{q}{ }_{i_{7}}{ }^{r} i_{10}{ }^{t}\left(2 \rho^{i_{1}}\left[i_{3} \mid i_{8} \rho^{i_{2}\left|i_{4}\right| i_{9}} \rho^{i_{5} i_{6} i_{7}} f_{i_{8} i_{9}} i_{10}\right]\right.
\end{aligned}
$$

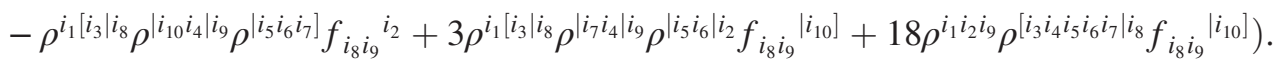

Now, using the identity

$$
\begin{aligned}
& \rho^{i_{1}\left[i_{3} \mid i_{8}\right.} \rho^{\left|i_{2} i_{4}\right| i_{9}} \rho^{\mid i_{5} i_{6} i_{7}} f_{i_{8} i_{9}}{ }^{\left.i_{10}\right]}=\frac{2}{7} \rho^{i_{1}\left[i_{3} \mid i_{8}\right.} \rho^{i_{2}\left|i_{4}\right| i_{9}} \rho^{\mid i_{5} i_{6} i_{7}} f_{i_{8} i_{9}}{ }^{\left.i_{10}\right]}-\frac{1}{7} \rho^{i_{1} i_{2} i_{8}} \rho^{\left[i_{3} i_{4} \mid i_{9}\right.} \rho^{\mid i_{5} i_{6} i_{7}} f_{i_{8} i_{9}}{ }^{\left.i_{10}\right]} \\
& -\frac{3}{7} \rho^{i_{1}\left[i_{3} \mid i_{8}\right.} \rho^{\left|i_{7} i_{4}\right| i_{9}} \rho^{\left|i_{5} i_{6}\right| i_{2}} f_{i_{8} i_{9}}{ }^{\left.\mid i_{10}\right]}-\frac{1}{7} \rho^{i_{1}\left[i_{3} \mid i_{8}\right.} \rho^{\left|i_{10} i_{4}\right| i_{9}} \rho^{\left.\mid i_{5} i_{6} i_{7}\right]} f_{i_{8} i_{9}}{ }^{i_{2}},
\end{aligned}
$$

for the first two terms in (B10), we obtain

$$
\begin{aligned}
& \left(\delta Z_{A B}{ }^{C}\right)_{3 \mathrm{rd}}=\frac{e \sqrt{5}}{5184 \sqrt{2}} d_{A B D} E_{k}{ }^{C} E_{l}{ }^{D} \epsilon_{m n p q r t} K_{i_{1}}{ }^{k}{ }_{i_{2}}{ }^{l} i_{3}{ }^{m}{ }_{i_{4}}{ }^{n}{ }_{i_{5}}{ }^{p}{ }_{i_{6}}{ }^{q} i_{i_{7}}{ }^{r}{ }_{i_{10}}{ }^{t}\left(7 \rho^{i_{1}\left[i_{3} \mid i_{8}\right.} \rho^{\left|i_{2} i_{4}\right| i_{9}} \rho^{\mid i_{5} i_{6} i_{7}} f_{i_{8} i_{9}}{ }^{\left.i_{10}\right]}\right. \\
& \left.+6 \rho^{i_{1}\left[i_{3} \mid i_{8}\right.} \rho^{\left|i_{7} i_{4}\right| i_{9}} \rho^{\left.\right|_{i} i_{6} \mid i_{2}} f_{i_{8} i_{9}}{ }^{\left.\mid i_{10}\right]}+\rho^{i_{1} i_{2} i_{8}} \rho^{\left[i_{3} i_{4} \mid i_{9}\right.} \rho^{i_{5} i_{6} i_{7}} f_{i_{8} i_{9}}{ }^{\left.i_{10}\right]}-18 \rho^{i_{1} i_{2} i_{8}} \rho^{\left[i_{3} i_{4} i_{5} i_{6} i_{7} \mid i_{9}\right.} f_{i_{8} i_{9}}{ }^{\left.\mid i_{10}\right]}\right) .
\end{aligned}
$$

Due to the high amount of index symmetries provided by contraction with the epsilon tensor, one is able to show that the first two terms are zero, whereas the third and the fourth can be rewritten in a fine form.

Indeed, start with the first term

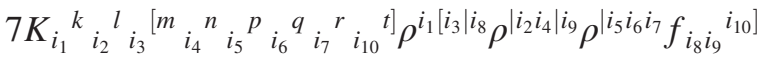

$$
\begin{aligned}
& =7 K_{i_{1}}{ }^{k}{ }_{i_{2}}{ }^{[l}{ }_{i_{3}}{ }^{m}{ }_{i_{4}}{ }^{n}{ }_{i_{5}}{ }^{p}{ }_{i_{6}}{ }^{q}{ }_{i_{7}}{ }^{r}{ }^{r}{ }_{10}{ }^{t} \rho^{i_{1} i_{3} i_{8}} \rho^{i_{2} i_{4} i_{9}} \rho^{i_{5} i_{6} i_{7}} f_{i_{8} i_{9}}{ }^{i_{10}}=0 \text {, }
\end{aligned}
$$

due to the antisymmetrization in seven indices $[$ lmnpqrt $]=0$ each running from 1 to 6 .

For the second term, we will use the generalized Yang-Baxter equation obtained from (B8), and the following identity

$$
\left.0=\rho^{\left[i_{5} i_{6} \mid i_{2}\right.} \rho^{\left|i_{3} i_{7} i_{4}\right| i_{9} i_{8} \mid i_{10}} f_{i_{8} i_{9}} i_{1}\right]=\frac{1}{7} \rho^{\left[i_{5} i_{6} \mid i_{2}\right.} \rho^{\left.\left|i_{3} i_{7} i_{4}\right| i_{9} i_{8} \mid i_{10}\right]} f_{i_{8} i_{9}}{ }^{i_{1}}-\frac{4}{7} \rho^{\left[i_{5} i_{6} \mid i_{2}\right.} \rho^{i_{1}\left|i_{7} i_{4}\right| i_{9} i_{8} \mid i_{10}} f_{i_{8} i_{9}}{ }^{\left.i_{3}\right]} .
$$

Taken together with the identity $\rho^{\left[i_{3} i_{6} \mid i_{8}\right.} \rho^{\mid i_{7} i_{4} i_{9}} f_{i_{8} i_{9}} i_{10}=0$ this implies

$$
\left.K_{i_{1} i_{2}\left[i_{3} i_{4} i_{5} i_{6} i_{7} i_{10}\right]}^{k l m n \rho_{5} \rho_{5} i_{6} i_{2}} \rho^{i_{3}\left[i_{1} \mid i_{8}\right.} \rho^{\left|i_{7} i_{4}\right| i_{9}} f_{i_{8} i_{9}}\right|_{\left.i_{10}\right]}=0 .
$$

Hence, we write for the second term in (B21) 


$$
\begin{aligned}
& 6 K_{i_{1}}{ }^{k} i_{2}{ }^{l}{ }^{l}{ }_{3}{ }^{[m}{ }_{i_{4}}{ }^{n}{ }_{i_{5}}{ }^{p}{ }_{i_{6}}{ }^{q}{ }_{i_{7}}{ }^{r} i_{10}{ }^{t} \rho^{\left[i_{5} i_{6} \mid i_{2}\right.} \rho^{i_{1}\left|i_{3}\right| i_{8}} \rho^{\left|i_{7} i_{4}\right| i_{9}} f_{i_{8} i_{9}}{ }^{\left.\mid i_{10}\right]} \\
& \stackrel{\text { gen.CYBE }}{=} 9 K_{i_{1}}{ }^{k}{ }_{i_{2}}{ }^{l} i_{3}{ }^{[m}{ }_{i_{4}}{ }^{n}{ }_{i_{5}}{ }^{p} i_{i_{6}}{ }^{q}{ }^{i_{7}}{ }^{r} i_{10}{ }^{t}{ }^{t} \rho^{\left[i_{5} i_{6} \mid i_{2}\right.} \rho^{\left.\left|i_{3} i_{7} i_{4}\right| i_{9} i_{8} \mid i_{10}\right]} f_{i_{8} i_{9} i_{1}}{ }^{i_{1}} \\
& =36 K_{i_{1}}{ }^{k}{ }_{i_{2}}{ }^{l}{ }_{i_{3}}{ }^{\left[m^{m}\right.}{ }_{i_{4}}{ }^{n}{ }_{i_{5}}{ }^{p}{ }_{i_{6}}{ }^{q}{ }^{q}{ }_{i_{7}}{ }^{r} i_{10}{ }^{t}{ }^{t]} \rho^{\left[i_{5} i_{6} \mid i_{2}\right.} \rho^{i_{1}\left|i_{7} i_{4}\right| i_{9} i_{8} \mid i_{10}} f_{i_{8} i_{9}}{ }^{\left.i_{3}\right]} \\
& =36 K_{i_{1}}{ }^{k} i_{2}{ }^{l}{ }^{l} i_{3}{ }^{[m}{ }_{i_{4}}{ }^{n}{ }_{i_{5}}{ }^{p}{ }_{i_{6}}{ }^{q}{ }_{i_{7}}{ }^{r} i_{10}{ }^{t} \rho^{i_{5} i_{6} i_{2}} \rho^{i_{1} i_{7} i_{4} i_{9} i_{8} i_{10}} f_{i_{8} i_{9}}{ }^{i_{3}} \\
& \stackrel{\text { genCYBE }}{=} 36 K_{i_{1}}{ }^{k}{ }_{i_{2}}{ }^{l}\left[i_{3}{ }^{[m} i_{i_{4}}{ }^{n}{ }^{n}{ }_{5}{ }^{p} i_{6}{ }^{q}{ }_{i_{7}}{ }^{r} i_{10}\right]{ }^{t} \rho^{i_{5} i_{6} i_{2}} \rho^{i_{3}\left[i_{1} \mid i_{8}\right.} \rho^{\left|i_{7} i_{4}\right| i_{9}} f_{i_{8} i_{9}}{ }^{\left.\mid i_{10}\right]}=0 \text {. }
\end{aligned}
$$

Where the last identity is due to (B15).

Next, we consider the third term in (B21). Again using the unimodularity condition and the identity $\rho^{\left[i_{3} i_{6} \mid i_{8}\right.} \rho^{\left.\mid i_{7} i_{4}\right] i_{9}} f_{i_{8} i_{9}} i_{10}=0$, one derives the following useful relation

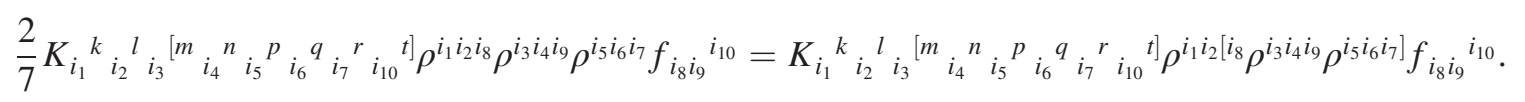

This allows rewriting the third term as

$$
K_{i_{1}}{ }^{k}{ }_{i_{2}}{ }^{l} i_{3}{ }^{[m}{ }_{i_{4}}{ }^{n}{ }_{i_{5}}{ }^{p}{ }_{i_{6}}{ }^{q}{ }_{i_{7}}{ }^{r} i_{10}{ }^{t]} \rho^{i_{1} i_{2} i_{8}} \rho^{\left[i_{3} i_{4} \mid i_{9}\right.} \rho^{\mid i_{5} i_{6} i_{7}} f_{i_{8} i_{9}}{ }^{\left.i_{10}\right]}=\frac{7}{2} K_{i_{1}}{ }^{k}{ }_{i_{2}}{ }^{l} i_{3}{ }^{[m}{ }_{i_{4}}{ }^{n}{ }_{i_{5}}{ }^{p}{ }_{i_{6}}{ }^{q}{ }_{i_{7}}{ }^{r}{ }_{i_{10}}{ }^{t]} \rho^{i_{1} i_{2}\left[i_{8}\right.} \rho^{i_{3} i_{4} i_{9}} \rho^{\left.i_{5} i_{6} i_{7}\right]} f_{i_{8} i_{9}}{ }^{i_{10}} \text {. }
$$

Finally, for the last term in (B21), we will need the identity

$$
\frac{2}{7} K_{i_{1}}{ }^{k}{ }_{i_{2}}{ }^{l}{ }_{i_{3}}{ }^{[m}{ }_{i_{4}}{ }^{n}{ }_{i_{5}}{ }^{p}{ }_{i_{6}}{ }^{q}{ }_{i_{7}}{ }^{r}{ }_{i_{10}}{ }^{t]} \rho^{i_{1} i_{2} i_{8}} \rho^{i_{3} i_{4} i_{5} i_{6} i_{7} i_{9}} f_{i_{8} i_{9}} i_{10}^{i_{10}}=K_{i_{1}}{ }^{k}{ }_{i_{2}}{ }^{l}{ }_{i_{3}}\left[{ }^{m}{ }_{i_{4}}{ }^{n}{ }_{i_{5}}{ }^{p}{ }_{i_{6}}{ }^{q}{ }_{i_{7}}{ }^{r} i_{i_{10}}{ }^{t]} \rho^{i_{1} i_{2}\left[i_{8}\right.} \rho^{\left.i_{3} i_{4} i_{5} i_{6} i_{7} i_{9}\right]} f_{i_{8} i_{9}}{ }^{i_{10}},\right.
$$

which is true upon the unimodularity constraint on $\rho^{i_{1} \ldots i_{6}}$. This gives for the fourth term

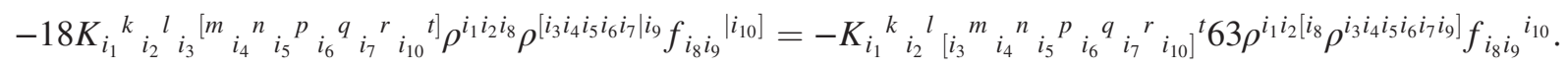

Altogether, the third order terms in the transformation of the Z-flux can be written as

$$
\begin{aligned}
& \delta\left(Z_{A B}{ }^{C}\right)_{3 \mathrm{rd}}=\frac{7 \sqrt{5} e}{10368 \sqrt{2}} e 6 d_{A B D} E_{k}{ }^{C} E_{l}{ }^{D} \epsilon_{m n p q r t} K_{i_{1}}{ }^{k}\left[i_{2}{ }^{l}{ }_{i_{3}}{ }^{m} i_{4}{ }^{n}{ }_{i_{5}}{ }^{p}{ }_{i_{6}}{ }^{q} i_{7}{ }^{r}{ }^{r}{ }_{10}{ }^{t}\right. \\
& \times\left(\rho^{i_{1} i_{2}\left[i_{8}\right.} \rho^{i_{3} i_{4} i_{9}} \rho^{\left.i_{5} i_{6} i_{7}\right]} f_{i_{8} i_{9}}{ }^{i_{10}}-18 \rho^{i_{1} i_{2}\left[i_{8}\right.} \rho^{\left.i_{3} i_{4} i_{5} i_{6} i_{7} i_{9}\right]} f_{i_{8} i_{9}}{ }^{i_{10}}\right) .
\end{aligned}
$$

We see that the terms are organized into the expression in brackets, which is antisymmetric in seven indices $i$ labeling Killing vectors. For group manifolds that have $i=1, \ldots, 6$, the third order terms would identically vanish. However, for general 6-dimensional manifolds with at least 7 Killing vectors, we find an additional constraint. Notably that the terms in brackets cannot, in general, be reduced to the generalized Yang-Baxter equation (47). Indeed, in the second term due to the antisymmetrization the indices $i_{8}, i_{9}$ stay either both on $\rho^{i_{1} \ldots i_{6}}$ or separate between $\rho^{i_{1} \ldots i_{3}}$ and $\rho^{i_{1} \ldots i_{6}}$. The latter case is evidently beyond something that can be massaged into (47).

[1] P. Townsend, Four lectures on M theory, in ICTP Summer School in High-Energy Physics and Cosmology (World Scientific, Singapore, 1997), pp. 385-438 [arXiv:hep-th/ 9612121].

[2] T. H. Buscher, A symmetry of the string background field equations, Phys. Lett. B 194, 59 (1987).

[3] T. H. Buscher, Path integral derivation of quantum duality in nonlinear sigma models, Phys. Lett. B 201, 466 (1988).
[4] X. C. de la Ossa and F. Quevedo, Duality symmetries from non-Abelian isometries in string theory, Nucl. Phys. B403, 377 (1993).

[5] C. Klimcik and P. Severa, Poisson-Lie T duality and loop groups of Drinfeld doubles, Phys. Lett. B 372, 65 (1996).

[6] C. Klimcik and P. Severa, Dual nonAbelian duality and the Drinfeld double, Phys. Lett. B 351, 455 (1995). 
[7] C. Klimcik, Poisson-lie T duality, Nucl. Phys. B, Proc. Suppl. 46, 116 (1996).

[8] C. Hull and P. Townsend, Unity of superstring dualities, Nucl. Phys. B438, 109 (1995).

[9] E. Witten, Some comments on string dynamics, in STRINGS 95: Future Perspectives in String Theory (World Scientific, Singapore, 1996), pp. 501-523 [arXiv:hep-th/9507121].

[10] Y. Sakatani, $U$-duality extension of Drinfel'd double, Prog. Theor. Exp. Phys. 2020, 023B08 (2020).

[11] E. Malek and D. C. Thompson, Poisson-lie U-duality in exceptional field theory, J. High Energy Phys. 04 (2020) 058.

[12] Y. Sakatani and S. Uehara, Non-Abelian $U$-duality for membranes, Prog. Theor. Exp. Phys. 2020, 073 B01 (2020).

[13] E. T. Musaev, On non-Abelian U-duality of $11 \mathrm{D}$ backgrounds, arXiv:2007.01213.

[14] Y. Sakatani, Extended Drinfel'd algebras and non-Abelian duality, arXiv:2009.04454.

[15] C. Cordova, T. T. Dumitrescu, and K. Intriligator, Deformations of superconformal theories, J. High Energy Phys. 11 (2016) 135.

[16] O. Lunin and J. M. Maldacena, Deforming field theories with $\mathrm{U}(1) \times \mathrm{U}(1)$ global symmetry and their gravity duals, J. High Energy Phys. 05 (2005) 033.

[17] E. Imeroni, On deformed gauge theories and their string/ M-theory duals, J. High Energy Phys. 08 (2008) 026.

[18] G. Arutyunov, R. Borsato, and S. Frolov, S-matrix for strings on $\eta$-deformed AdS5 x S5, J. High Energy Phys. 04 (2014) 002.

[19] B. Hoare and F. K. Seibold, Supergravity backgrounds of the $\eta$-deformed $\mathrm{AdS}_{2} \times S^{2} \times T^{6}$ and $\mathrm{AdS}_{5} \times S^{5}$ superstrings, J. High Energy Phys. 01 (2019) 125.

[20] G. Arutyunov, R. Borsato, and S. Frolov, Puzzles of $\eta$-deformed $\mathrm{AdS}_{5} \times \mathrm{S}^{5}$, J. High Energy Phys. 12 (2015) 049.

[21] G. Arutyunov, S. Frolov, B. Hoare, R. Roiban, and A. A. Tseytlin, Scale invariance of the $\eta$-deformed $\mathrm{AdS}_{5} \times S^{5}$ superstring, T-duality and modified type II equations, Nucl. Phys. B903, 262 (2016).

[22] B. Hoare and A. A. Tseytlin, Type IIB supergravity solution for the T-dual of the $\eta$-deformed $\mathrm{AdS}_{5} \times \mathrm{S}^{5}$ superstring, J. High Energy Phys. 10 (2015) 060.

[23] L. Wulff and A. A. Tseytlin, Kappa-symmetry of superstring sigma model and generalized 10d supergravity equations, J. High Energy Phys. 06 (2016) 174.

[24] T. Araujo, I. Bakhmatov, E. Ó Colgáin, J. Sakamoto, M. M. Sheikh-Jabbari, and K. Yoshida, Yang-Baxter $\sigma$-models, conformal twists, and noncommutative Yang-Mills theory, Phys. Rev. D 95, 105006 (2017).

[25] T. Araujo, I. Bakhmatov, E. O. Colgáin, J.-i. Sakamoto, M. M. Sheikh-Jabbari, and K. Yoshida, Conformal twists, Yang-Baxter $\sigma$-models \& holographic noncommutativity, J. Phys. A 51, 235401 (2018).

[26] R. Borsato and L. Wulff, Non-Abelian T-duality and YangBaxter deformations of Green-Schwarz strings, J. High Energy Phys. 08 (2018) 027.

[27] I. Bakhmatov, O. Kelekci, E. Ó Colgáin, and M. M. SheikhJabbari, Classical Yang-Baxter equation from supergravity, Phys. Rev. D 98, 021901 (2018).
[28] I. Bakhmatov and E. T. Musaev, Classical Yang-Baxter equation from $\beta$-supergravity, J. High Energy Phys. 01 (2019) 140.

[29] I. Bakhmatov, E. Ó Colgáin, M. M. Sheikh-Jabbari, and H. Yavartanoo, Yang-Baxter deformations beyond coset spaces (a slick way to do TsT), J. High Energy Phys. 06 (2018) 161.

[30] O. Hohm, C. Hull, and B. Zwiebach, Background independent action for double field theory, J. High Energy Phys. 07 (2010) 016.

[31] O. Hohm, C. Hull, and B. Zwiebach, Generalized metric formulation of double field theory, J. High Energy Phys. 08 (2010) 008.

[32] I. Bakhmatov, N. S. Deger, E. T. Musaev, E. O. Colgáin, and M. M. Sheikh-Jabbari, Tri-vector deformations in $d=11$ supergravity, J. High Energy Phys. 08 (2019) 126.

[33] D. S. Berman, M. Cederwall, U. Gran, H. Larsson, M. Nielsen, B. E. Nilsson, and P. Sundell, Deformation independent open brane metrics and generalized theta parameters, J. High Energy Phys. 02 (2002) 012.

[34] I. Bakhmatov, K. Gubarev, and E. T. Musaev, Non-abelian tri-vector deformations in $d=11$ supergravity, J. High Energy Phys. 05 (2020) 113.

[35] E. Malek, Y. Sakatani, and D. C. Thompson, E6(6) exceptional Drinfel'd algebras, J. High Energy Phys. 01 (2021) 020 .

[36] J.-i. Sakamoto, Y. Sakatani, and K. Yoshida, Homogeneous Yang-Baxter deformations as generalized diffeomorphisms, J. Phys. A 50, 415401 (2017).

[37] A. Çatal Özer and S. Tunalı, Yang-Baxter deformation as an $O(d, d)$ transformation, Classical Quantum Gravity 37, 075003 (2020).

[38] O. Hohm and H. Samtleben, Exceptional field theory I: $E_{6(6)}$ covariant form of M-theory and Type IIB, Phys. Rev. D 89, 066016 (2014).

[39] E. Musaev and H. Samtleben, Fermions and supersymmetry in $\mathrm{E}_{6(6)}$ exceptional field theory, J. High Energy Phys. 03 (2015) 027.

[40] A. Baguet, O. Hohm, and H. Samtleben, $\mathrm{E}_{6(6)}$ exceptional field theory: Review and embedding of Type IIB, in Proceedings, 14th Hellenic School and Workshops on Elementary Particle Physics and Gravity (CORFU2014), Proc. Sci., CORFU2014 (2015) 133 [arXiv:1506.01065].

[41] D. S. Berman and C. D. Blair, The geometry, branes and applications of exceptional field theory, Int. J. Mod. Phys. A 35, 2030014 (2020).

[42] E. T. Musaev, U-dualities in type II and M-theory: A covariant approach, Symmetry 11, 993 (2019).

[43] E. Cremmer, B. Julia, H. Lu, and C. Pope, Dualization of dualities. 1, Nucl. Phys. B523, 73 (1998).

[44] C. D. A. Blair and E. Malek, Geometry and fluxes of SL(5) exceptional field theory, J. High Energy Phys. 03 (2015) 144.

[45] D. S. Berman, H. Godazgar, M. J. Perry, and P. West, Duality invariant actions and generalised geometry, J. High Energy Phys. 02 (2012) 108.

[46] E. T. Musaev, Gauged supergravities in 5 and 6 dimensions from generalised Scherk-Schwarz reductions, J. High Energy Phys. 05 (2013) 161. 
[47] R. Borsato, A. Vilar Lopez, and L. Wulff, The first $\alpha^{\prime}$-correction to homogeneous Yang-Baxter deformations using $O(d, d)$, J. High Energy Phys. 07 (2020) 103.

[48] B. de Wit, H. Samtleben, and M. Trigiante, The maximal $D=5$ supergravities, Nucl. Phys. B716, 215 (2005).

[49] A. Le Diffon and H. Samtleben, Supergravities without an action: Gauging the trombone, Nucl. Phys. B811, 1 (2009).

[50] F. Riccioni, D. Steele, and P. West, The E(11) origin of all maximal supergravities: The hierarchy of field-strengths, J. High Energy Phys. 09 (2009) 095.

[51] D. Andriot and A. Betz, $\beta$-supergravity: A ten-dimensional theory with non-geometric fluxes, and its geometric framework, J. High Energy Phys. 12 (2013) 083.

[52] D. S. Berman and M. J. Perry, Generalized geometry and M theory, J. High Energy Phys. 06 (2011) 074.
[53] E. T. Musaev, Exceptional field theory: $S L(5)$, J. High Energy Phys. 02 (2016) 012.

[54] D. S. Berman, E. T. Musaev, and D. C. Thompson, Duality invariant M-theory: Gauged supergravities and ScherkSchwarz reductions, J. High Energy Phys. 10 (2012) 174.

[55] K. Peeters, A field-theory motivated approach to symbolic computer algebra, Comput. Phys. Commun. 176, 550 (2007).

[56] K. Peeters, Introducing cadabra: A symbolic computer algebra system for field theory problems, arXiv:hep-th/ 0701238 .

[57] K. Peeters, Cadabra2: Computer algebra for field theory revisited, J. Open Source Softw. 3, 1118 (2018).

[58] K. Gubarev and E. T. Musaev, Github repository, http:// github.com/emusaev/genYB_E6 (2020). 